\title{
DAL PLURALISMO POLARIZZATO \\ AL PLURALISMO MODERATO. IL MODELLO \\ DI SARTORI E LA TRANSIZIONE ITALIANA
}

\author{
di Adriano Pappalardo
}

\section{Introduzione}

Negli anni novanta, l'Italia è entrata in un processo di transizione che è ormai divenuto oggetto di un'abbondante letteratura nazionale e straniera ${ }^{1}$. Piuttosto ovviamente, la natura di tale transizione è ben diversa da quelle dei paesi postcomunisti e, come ha opportunamente sottolineato Pasquino (1994; 1995), poco comparabile con altri precedenti storici. Anche la Francia, che questo autore considera il miglior termine di confronto, lo è in realtà assai relativamente sotto una varietà di punti di vista. Mentre infatti la Quarta e, per lungo tempo, anche la Quinta Repubblica sono rimasti classici casi di pluralismo polarizzato (Sartori 1982, 256-262), l'Italia degli anni novanta non può più essere definita tale, e proprio per questo (o, almeno, anche per questo) si è avviata alla presente transizione. Come è noto, inoltre, le riforme golliste investirono essenzialmente le principali istituzioni politiche della repubblica, ma lasciarono inalterati il subsistema burocratico e la struttura (centralizzata) dello Stato, che sono, invece, componenti tutt'altro che secondarie per comprendere il decorso e gli eventuali sbocchi della crisi italiana. Ma, infine, tale crisi si intreccia anche al declino dello Stato sociale e interventista, coinvolgendo la ridefinizione dei confini fra politica ed economia e il ruolo delle grandi organizzazioni degli interessi, sindacati in testa. Come dire che, oltre al sistema politico-istituzionale ed alla pubblica amministrazione, le rela-

Ringrazio Antonio Agosta, Aldo Di Virgilio, Renato Mannbeimer, Oreste Massari e Leonardo Morlino per avermi cortesemente messo a disposizione molti dei dati elaborati in questo articolo.

1 Oltre ai lavori citati nel testo, vedi Calvi e Vannucci (1995), Diamanti e Mannheimer (1994), Ignazi e Katz (1995).

RIVISTA ITALIANA DI SCIENZA POLITICA / a. XXVI, n. 1, aprile 1996 
zioni industriali e i loro attori pubblici e privati rappresentano una terza dimensione di cambiamento, che altrove è stata poco importante (Francia), ovvero incomparabilmente diversa (regimi postcomunisti).

Tutto questo non significa negare che i processi di cambiamento del sistema partitico, l'evoluzione istituzionale e il ricambio della classe politica in Italia «presentano una quantità di somiglianze con problemi affrontati nel caso francese» (Pasquino 1994, 3). Ma, per un verso, bisogna vedere quante somiglianze, una volta messe in conto le differenze contestuali ricordate e altre ancora. E, per altro verso, è discutibile se somiglianze e differenze possano essere correttamente comparate fra sistemi che hanno completato le loro transizioni e sistemi nei quali esse sono tuttora in corso. Normalmente, infatti, quando si citano la Francia (o anche la Spagna o la Germania) si pongono in rapporto attori, meccanismi elettorali, o altre istituzioni più o meno consolidate, con corrispondenti tendenze che consolidate non sono, se è vero che un gran numero di osservatori conviene che la transizione italiana è «difficile e confusa» (D'Alimonte e Bartolini 1995, 466), «ambigua e con non poche incognite» (Ceccanti e Fabbrini 1995, 281), dominata dall'incertezza (Morlino 1995) e, detto per esteso, complessa, lunga, indeterminata, reversibile (Pasquino 1995).

Anche se non li condivido del tutto, questi giudizi invitano evidentemente alla cautela nelle comparazioni o nelle inferenze. E una strategia cautelativa - secondo me fruttosa quanto trascurata - consiste nell'ancorare l'analisi della transizione a una salda base teorica, cioè ai modelli già proposti per spiegare il sistema politico italiano, e, eventualmente, in grado di indicarne direzione e stadio di evoluzione. Con le altre possibili, questa strategia condivide l'oggetto, poiché si tratta comunque di chiedersi da dove venga il sistema politico, perché, in che cosa e fino a che punto sia cambiato e dove potrebbe condurre il cambiamento. $\mathrm{Ma}$ rispetto ai chi risponde basandosi su precedenti empirici più o meno eterogenei o sugli esiti consolidati di questa o quella transizione storica, scegliere e seguire accuratamente un modello di riferimento è un forte incentivo alla disciplina espositiva e al rigore dei ragionamenti. $\mathrm{Da}$ un lato, infatti, i modelli - se ben costruiti e sufficientemente articolati - forniscono una guida sistematica, e teoricamente giustificata, che per definizione manca ai troppi resoconti «narrativi», comparati o no, circolanti sulla transizione. E, in secon- 
do luogo, disporre di un modello è acquisire un parametro, cioè fissare l'indispensabile punto fermo solo in rapporto al quale ha senso parlare di cambiamento, della sua direzione e della sua profondità. Nel prossimo paragrafo, pertanto, procederò a specificare questo parametro, per passare, poi, alla sua applicazione al caso italiano.

\section{Modelli di democrazia}

Le transizioni politiche sono processi, più o meno complessi, di cambiamento da uno stato precedente, più o meno consolidato, ad uno successivo, da raggiungere in tempi, con modalità ed esiti più o meno difficilmente prevedibili. Nelle transizioni, infatti, coesistono tipicamente elementi di continuità con il passato ed innovazioni, e la mistura degli uni e delle altre è per definizione variabile, la variazione spesso rapida e le sue direzioni ambigue o anche contraddittorie. Si capisce, perciò, che gli osservatori tendano a rifugiarsi nell'incertezza, o concludano modestamente che «gran parte della posta dipende dalle decisioni o non decisioni che i leader dei partiti saranno capaci di prendere» (Morlino 1995, 23). Ovviamente, però, le élites stesse sono condizionate da vincoli ed opportunità strutturali o, per ripetermi, da continuità con, o innovazioni dallo stato precedente del sistema. Di conseguenza, tale stato o, meglio, le sue concettualizzazioni, i modelli teorici ed i dati pertinenti disponibili, sono, in generale, i parametri obbligati ai quali commisurare i processi di transizione o, perlomeno, dai quali cominciarne l'esame. Ma se la raccomandazione appare abbastanza scontata, le applicazioni diligenti lo sono assai meno e, per esempio, anche i (pochi) seri contributi sull'Italia fanno riferimenti per lo più sommari e rituali ai modelli usati per analizzare la cosiddetta «Prima Repubblica». Nella migliore delle ipotesi, questi modelli vengono ricordati per «situare» il caso o, come direbbe Sartori $(1976,287)$, «per tracciare una carta, una mappa». Ma sebbene l'uso sia legittimo ed altamente informativo, fermarsi ad esso significa amputare le capacità esplicative e predittive dei modelli, rinunciare a leggerli come una sequenza di cause e conseguenze logicamente interconnesse e più o meno rilevanti empiricamente (Idem, 287-290) e, dunque, precludersi precisamente un esame corretto e disciplinato dei pochi o tanti vincoli (ma anche opportunità) che le concettualizzazioni dello stato 
del sistema pongono ai (o escludono dai) suoi attuali (ed, eventualmente, futuri) processi di cambiamento.

Il risultato è approssimatezza e confusione, alle quali, peraltro, non contribuiscono solo sottovalutazioni, o fraintendimenti, di un dato modello causale, delle sue variabili, delle relazioni fra loro e/o delle conseguenti implicazioni. Talvolta, come vedremo, definizioni e relazioni fra variabili chiave sono diverse per diversi autori, che scelgono - esplicitamente o implicitamente - modelli diversi, o adottano soluzioni operazionali che fanno differenza per il giudizio sul decorso e gli esiti prevedibili della transizione. A maggior ragione, vale la pena di fare uno sforzo di chiarificazione e di pulizia analitica che può essere guidato, propongo, dalla discussione della figura 1 .

La figura riporta, con qualche modificazione, la tipologia dei sistemi partitici di Sartori (1976, 288-289), insieme all'«informazione rilevante che essa fornisce riguardo al sistema politico nel suo complesso» (286). Come è noto, tipologia e associate proprietà sistemiche sono un obbligato punto di riferimento della letteratura politologica e in particolare di quella sull'Italia, storicamente uno dei principali casi di pluralismo polarizzato. Ma dovrebbe essere ormai chiaro che, in questo paragrafo, più del caso interessa che cosa il framework spiega e predice esattamente, con quali concetti, secondo quali sequenze causali e con quali conclusioni in merito agli stati ed, eventualmente, alle dinamiche dei regimi democratici. Nella figura, la sequenza è aperta da un indicatore di frammentazione del sistema, espressa in termini di numero di partiti. I partiti, a loro volta, sono individuati dalle celebri regole di conteggio di Sartori (potenziale di coalizione e potenziale di intimidazione), ma - e questo è un primo punto di solito sfuggito agli osservatori - il loro utilizzo è subordinato alla verifica di due condizioni di applicabilità. La prima è che, per entrare nel calcolo della frammentazione, un partito deve essere autenticamente tale non una «mera etichetta, una coalizione sfilacciata di notabili spesso cangiante ad ogni elezione e a rischio di dissolversi da un'elezione all'altra» (Sartori 1976, 284 e 349). Partiti, insomma, sono considerati solo le formazioni strutturate, cioè consolidate, perlomeno quanto basta a distinguerle da una situazione di «atomizzazione»; e - seconda condizione - partiti strutturati fanno un sistema, con peculiari proprietà, se, e solo se, interagiscono nello stesso spazio competitivo, mentre sono irrilevanti se se ne stanno isolati su dimensioni diverse, con il sostegno sicuro di 
画是

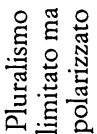

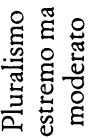

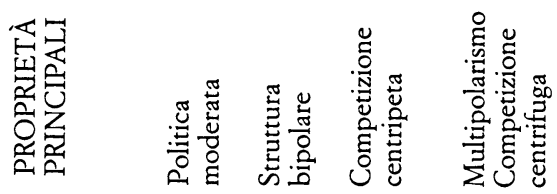

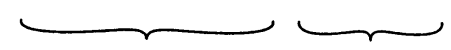

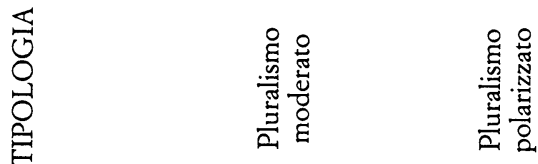

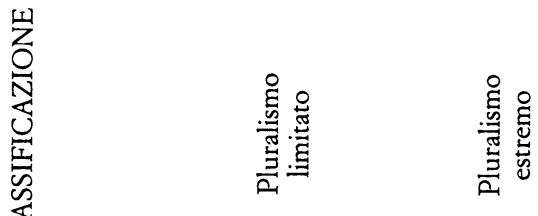

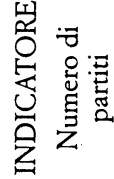

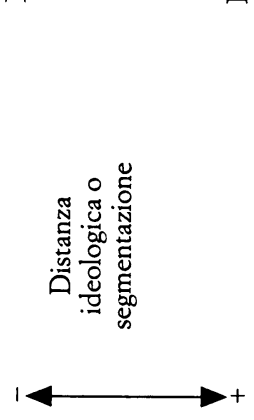

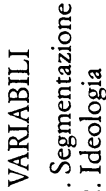

$n$

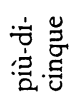

$\dot{a}$
$\stackrel{\infty}{\sim}$
$\dot{\infty}$
$\stackrel{\infty}{\sim}$
$\dot{0}$
$\stackrel{2}{2}$

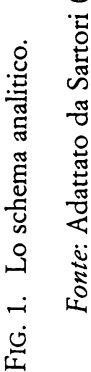


un elettorato identificato, ma senza capacità espansive e, come tali, «fuori dalla corsa» cui partecipano tutti gli altri ${ }^{2}$.

Come vedremo, le conseguenze delle due specificazioni per la diagnosi della transizione italiana sono tutt'altro che irrilevanti, direttamente e indirettamente. Direttamente, infatti, esse permetteranno di determinare il formato del sistema partitico con più precisione di quanto sia stato fatto finora $e$, di conseguenza, di individuarne le «predisposizioni meccaniche», gli attributi funzionali dai quali dipende come il sistema «lavora». Dico di conseguenza perché il numero è un indicatore potente (seppur non infallibile) di queste predisposizioni e, per cominciare, della distanza ideologica, indicata come variabile interveniente nella seconda colonna della figura. Il punto, cogentemente argomentato da Sartori ed empiricamente corroborato da più di una fonte ${ }^{3}$, non richiederebbe, in sé, altri commenti in questa sede, ma poiché le sue applicazioni ed implicazioni sono spesso equivocate o contestate, conviene ricordarlo per esteso insieme alle une e alle altre.

Nella formulazione classica, «l'assunto fondato sull'evidenza è che l'esistenza di diversi partiti rilevanti è correlata con un più esteso spazio competitivo» e che, oltre una soglia critica (più-di-cinque), l'estensione in parola è prevedibilmente massima. Ciò implica che vari sistemi «esibiscono differenti distanze lineari complessive», le quali esprimono convenientemente in termini spaziali unidimensionali «le altrimenti ben note distinzioni fra culture politiche omogenee ed eterogenee, società consensuali o conflittuali ... sistemi non polarizzati e polarizzati». In principio queste distinzioni possono essere registrate su diverse dimensioni, fermo restando che c'è polarizzazione ogni qual volta le forze politiche e gli elettorati occupanti gli estremi dello spazio «si trovano così distanti da formare due poli opposti», espressione di «una dispersione massima dell'opinione». $\mathrm{Ma}$ la frammentazione è particolarmente importante perché, come ricordato, essa segnala di regola la polarizzazione sulla dimensione ideologica sinistra-destra, ossia la presenza di «due poli opposti antisistema», che pongono in questione la legittimi-

2 Sartori (1976, 339-342 e 348-349). Tali partiti, in altre parole, sono rilevanti in termini di «dominî di identificazione» nel sistema, ma non quanto alla loro «dimensione di competizione». La distinzione sartoriana è al centro della ricerca di Bartolini e Mair (1990) e su di essa tornerò in maggior dettaglio nel prossimo paragrafo.

3 Sartori (1976, 283-293); Sani e Sartori (1978); Bartolini e Mair (1990, cap. 8). 
tà stessa della democrazia; e non solo questa è la dimensione logicamente e di fatto dominante nelle società occidentali; ma è anche l'unica la cui polarizzazione abbia conseguenze di rilievo per la competizione politica e per il rendimento complessivo dei sistemi interessati ${ }^{4}$.

Prima di passare alle conseguenze, però, occorre esaminare una controversia suscitata da questo argomento, che può offuscarne il potenziale e il contenuto esplicativo e predittivo. La controversia concerne la struttura dello spazio competitivo, che non tutti considerano «elastico» (cioè, di estensione variabile) e necessariamente unidimensionale (o lineare). Per qualcuno, al contrario, il concetto di elasticità è irrilevante, o anche fuorviante, mentre lo spazio può essere sia unidimensionale che multidimensionale, ed è questa la differenza che conta per capire come «lavorano» i sistemi politici.

Nei termini di D'Alimonte (1978), che anticipano concetti sui quali tornerò, democrazie «competitive» ed «efficienti» presuppongono infatti uno spazio lineare e «l'elasticità del voto delle minoranze critiche». Correlativamente, efficienza e competizione sono da escludere se due o più dimensioni sono politicamente salienti e ordinate lessicograficamente dagli elettori; e nella seconda categoria, appunto, rientrano i sistemi polarizzati, cioè gli stessi che Sartori associa a uno spazio «stirato». Come dire che i due approcci si dividono sull'explanans e, di conseguenza, sul raggruppamento dei casi empirici: se, infatti, D'Alimonte può attribuire allo stesso tipo Italia, Austria, Belgio, Olanda e Svizzera $(1978,472)$ perché tutti sistemi a più dimensioni ordinate lessicograficamente, nello spazio lineare elastico il primo paese si distingue per una distanza ideologica assai maggiore. E poiché sappiamo che diverse distanze sono funzione del numero dei partiti, va da sé che a questo approda l'intera controversia: se il numero conti (Sartori), ovvero non conti, come sottintende D'Alimonte mettendo assieme paesi di tutti i possibili formati.

Una volta fatta questa scelta, però, molte altre seguono a cascata. Poiché il formato è irrilevante, infatti, non ha senso preoccuparsi di determinarlo correttamente, cioè di ricorrere alle due regole di conteggio di Sartori; a fortiori, non ha senso delimitare l'ambito entro cui contare, distinguendo fra partiti 
«veri» (strutturati) e partiti atomizzati; ed è addirittura inammissibile limitare l'applicazione delle regole ai partiti in «competizione sulla stessa ed unica dimensione» (Sartori 1976, 349) perché nel modello di D'Alimonte è proprio l'interazione fra dimensioni che interessa e, quindi, tutti i partiti che ne registrino/ attivino almeno una.

In breve, la controversia è di grande portata e, naturalmente, tale rimane indipendentemente dal fatto che $\mathrm{i}$ due modelli siano applicati a sistemi consolidati o in transizione: tanto è vero che, nel recente saggio scritto con Bartolini sulle tendenze del sistema partitico italiano, D'Alimonte $(1955,439-443)$ si occupa estesamente del formato uscito dalle elezioni del 1994, ma senza discriminare fra partiti rilevanti e irrilevanti; non dedica alcuna discussione alla peculiare posizione della Lega Nord, in parte attestata sulla dimensione sinistra-destra, in parte solitariamente «alienata» sulla dimensione centro-periferia; ed esamina le nuove dinamiche competitive del sistema in termini di scostamenti in corso dal modello multidimensionale e dalle preferenze lessicografiche storiche dell'elettorato. Ne risulta una trattazione tecnicamente sofisticata e ricca di informazioni, ma pur sempre orientata da premesse teoriche che si riflettono inevitabilmente sulla scelta delle variabili rilevanti e sulle spiegazioni e predizioni formulate. Poiché in questo paragrafo interessa il modello da applicare, dunque, è d'obbligo chiedersi se scelta e spiegazione sono davvero giustificate, cioè se reggono il confronto con il modello unidimensionale. E la risposta - a mio avviso inequivocabile - è no, per diversi buoni motivi 5 .

Il primo motivo è lo status logico ed empirico comparativamente solido dell'ipotesi dello spazio lineare «elastico», al quale contribuiscono gli argomenti di Sartori e le prove fornite insieme a Sani e, indipendentemente, da altri autori ${ }^{6}$. Ammesso che questo non basti, un'altra considerazione concerne la parsimonia dei due modelli: due o più dimensioni, ordinate lessicograficamente o meno, formano infatti un complesso di assunti meno «economico» di una sola, e sarebbero un'alternativa preferibile solo se spiegassero meglio e di più. $\mathrm{Ma}$ - terzo - così non è, in quanto l'obiettivo finale di entrambi i modelli è spiegare e predire il rendimento (stabilità ed efficienza) dei regimi democratici; e ho già ricordato che D'Alimonte mette l'Italia assieme al-

5 Gli argomenti che seguono sono approfonditi in Pappalardo (1989, 210-215).

6 Vedi la nota 3. 
l'Olanda e in generale ai sistemi segmentati, aggregando non solo formati, ma anche rendimenti diversissimi ${ }^{7}$.

Il modello unidimensionale, per contro, discrimina con maggior aderenza ai dati e utilizzando il criterio numerico - da solo o, se non risolutivo, con la distanza ideologica - assegna notoriamente l'Italia (degli anni settanta) al pluralismo polarizzato e i paesi ad alta segmentazione, ma bassa o limitata polarizzazione, al pluralismo moderato. Siamo così alla quarta colonna della figura 1, a fianco della quale vengono specificate le principali «predisposizioni meccaniche» associate ai tipi, per noi della massima importanza in quanto è nel superamento della polarizzazione e nel passaggio al pluralismo moderato che, in pratica, sono individuati i nodi della transizione italiana e su questi nodi se ne valuta lo stadio evolutivo e se ne anticipano le prospettive. $\mathrm{Ma}$, ancora una volta, valutazioni e previsioni prescindono dalla logica del modello di cui prendono a prestito concetti chiave e vanno, intenzionalmente o meno, per proprio conto. Prendiamo la nozione di competizione che, appunto, assume differenze critiche fra tipi di sistema partitico e, a maggior ragione, per la transizione da un tipo all'altro. Al riguardo, Sartori sottolinea che i concetti dei quali tener conto sono in realtà due (direzione della competizione e competitività), una questione sulla quale la letteratura è divisa ${ }^{8}$. Esplicitamente o implicitamente, infatti, D'Alimonte e Bartolini (1995) rifiutano la distinzione e conducono la loro analisi solo in termini di competitività. $\mathrm{Ma}$, così facendo, essi compiono una scelta che merita un ampio commento, poiché le sue implicazioni sono veramente cruciali.

Per cogliere queste implicazioni si può cominciare col ricordare che per Sartori $(1976,218-220,293)$ «la competitività è un particolare stato del gioco», in quanto tale «da misurare dal risultato, dalla sua effettività». In altre parole, «solo i risultati elettorali possono dimostrare fino a che punto un sistema è competitivo», ma se e quale significato abbia qualunque conclusione in proposito non può essere stabilito dal «crudo operazionismo» di una (o più) misure di competitività. Per questo bisogna spostarsi, appunto, sulla direzione della competizione, il cui accertamento è decisivo per diversi motivi.

7 Lijphart (1968; 1968a).

8 La divisione di cui si parla nel testo non va confusa con quella su cui è incentrato un altro lavoro di D'Alimonte (1989), che non è pertinente in questa sede. 
Il primo motivo è di portata metodologica generale e sottintende la ben nota strategia di ricerca sartoriana fondata sulla precedenza dell'analisi qualitativa sull'analisi quantitativa, del trattamento discontinuo sul trattamento continuo dei concetti (1976, 293-299). Se adattata ai problemi che ci interessano, infatti, questa strategia suggerisce che una variabile continua (competitività) non è appropriata a cogliere proprietà discontinue eventualmente attribuibili al pluralismo polarizzato e al pluralismo moderato. $\mathrm{E}$ poiché, in effetti, la direzione della competizione è stipulata discontinua (centrifuga o centripeta) nei due tipi, non si può non tenerne conto in uno schema interpretativo teoricamente informato. Altrimenti - ammonisce Sartori - si fa «crudo operazionismo», che non solo genera appiattimento (di differenze qualitative) e conseguente perdita o distorsione di informazione; ma, nel caso interessato, tende a sostituire appiattimento e post-dizione a potere discriminante, spiegazione e predizione.

Di queste trappole, mi sembra cadano vittima D'Alimonte e Bartolini, che si propongono di valutare il passaggio dell'Italia a un tipo di sistema partitico più competitivo; ma poiché riducono la «struttura della competizione» alle sue misure (volatilità, margini di vittoria nei collegi, sommabilità degli elettori dei due principali schieramenti), finiscono con il dimenticare $i$ «salti», o le «soglie», inerenti alla logica classificatoria e, dunque, ai tipi e alle loro proprietà'. Quel che resta sono così differenze di grado, tanto precise quanto sprovviste proprio del necessario potere discriminante. Una volta eretta la misurazione (cioè, la competitività) a criterio, infatti, quale confine potrà essere posto alla logica del «più o meno»? E quando un qualsiasi indicatore continuo segnalerà il punto di svolta da un sistema non competitivo (pluralismo polarizzato) ad un sistema competitivo (pluralismo moderato)? Quando - rispondono D'Alimonte e Bartolini (1995, 453-454) - «l'elettorato mobile sia decisivo non solo ai fini della distribuzione dei seggi fra i partiti, ma dell'assegnazione della maggioranza dei seggi tra schieramenti in diretta competizione tra loro per la formazione del governo».

Il termine chiave «decisivo», a sua volta, è definito dalla

9 D'Alimonte e Bartolini (1995); Bartolini e D'Alimonte (1995). Ciò non toglie che delle misure dei due autori si debba discutere, e si discuterà ampiamente, nel prossimo paragrafo. 
«consistenza» o, per D'Alimonte, anche dalla collocazione spaziale del voto fluttuante ${ }^{10}$, e come tale rinvia ad un interrogativo semplice e ad una risposta altrettanto disarmante. L'interrogativo è: «Quanti elettori fanno la differenza?». O, in altre parole, «quanti dovrebbero essere gli elettori disponibili per poterli dire decisivi?». E la risposta è: «A priori, non si può dire» (D'Alimonte 1989, 315), o - come sosteneva Sartori - dipende dai risultati elettorali, anzi (se «decisiva» è «l'assegnazione della maggioranza»), dipende dal fatto che ci sia stata o meno rotazione al potere tra schieramenti contrapposti.

Come volevasi dimostrare, la conclusione raggiunta è postdittiva, contingente all'osservazione (elezione) di volta in volta interessata ${ }^{11}$. La perdita di prospettiva corrispondente è tanto evidente da rafforzare il dubbio che il rigore quantitativo sia la via maestra per valutare correttamente i processi di transizione. $\mathrm{Ma}$, se non bastasse, a sfavore della concentrazione sulla competitività, e a favore dell'analisi della direzione della competizione, depongono altri argomenti, in parte riconosciuti dagli stessi autori che sto criticando. Il primo è che molte delle misure in circolazione sono discutibili o utilizzate discutibilmente (Bartolini 1986; 1995); secondariamente, il rigore di questa o quella misura fa posto all'incertezza o all'ambiguità quando più misure si contendono il campo con risultati diversi o contrastanti ${ }^{12}$; infine, la questione cruciale è che l'interesse dell'intero dibattito sulla competizione dipende dalla differenza che essa fa per l'«efficienza» della democrazia, cioè per la ricettività dei go-

10 D'Alimonte $(1989,315)$. Bartolini $(1995,22)$ lascia invece impregiudicata la questione della collocazione spaziale.

11 In un successivo lavoro, Bartolini $(1995,20-22)$ riconosce il problema e abbandona la nozione di «decisività delle elezioni» per una misura di competitività (o «vulnerabilità») meno vincolata all'effettivo risultato elettorale e all'alternanza al potere. Ma, sebbene assai più sofisticata, la sua soluzione non soddisfa del tutto e rimane comunque esposta alle altre obiezioni formulate nel testo. In particolare, essendo basata sul rapporto fra margine di vittoria in una data elezione e volatilità media nelle precedenti, la misura - riconosce lo stesso Bartolini (9-12) - esprime solo una parte della «disponibilità» elettorale totale presente nel sistema. E poiché su quest'ultima non possediamo «informazioni precise» (cioè, quantificabili), la sua scelta è di sottostimarla, mentre ciò che io sto perseguendo nel testo, e ciò a cui mi atterrò in seguito, è la strategia sartoriana di «porre gli sviluppi quantitativi entro le direttrici dell'analisi qualitativa» $(1976,299)$, anche nell'intento di segnalare errori di sotto-estimazione e, possibilmente, di indicare come controllarli.

12 Un esempio pertinente alla transizione italiana è Melchionda (1995) le cui misure sono, appunto, in contrasto con quelle di Bartolini e D'Alimonte. Sulla questione ritorno nel prossimo paragrafo. 
vernanti nei confronti dei governati (Sartori 1977; D'Alimonte 1978); e in questo la competitività è di poco o punto giovamento, non solo perché le sue «conseguenze sistemiche non sono affatto chiare» (Sartori 1976, 293), ma anche perché la massimizzazione dell'accoppiata competitività/ricettività può tradursi in policy outputs crescentemente inefficienti, nel caos politico o, perfino, nella crisi e nel crollo dei sistemi democratici.

Il punto, già ricordato da Sartori $(1976,219)$ e ammesso in qualche misura da D'Alimonte (1989, 316-318), è sottolineato con forza da Bartolini e altri ${ }^{13}$, e autorizza ovvie conclusioni. A quanto pare, non solo la competitività fornisce una bussola deformata e parziale per la classificazione o riclassificazione dei sistemi politici, se e in quanto prescinde dalla direzione della competizione; ma la bussola è anche impazzita, può condurre da molte parti, cogliere proprietà o differenze sistemiche di volta in volta variabili o contraddittorie, funzionali e disfunzionali o, eventualmente, rivelarsi incapace di «produrre differenze purchessia nel comportamento delle élites o nel rendimento dei governi» (Sartori 1976, 293). Dal punto di vista descrittivo, la nozione si conferma dunque virtualmente inservibile, se prescelta come variabile indipendente o variabile esplicativa chiave. Conformemente, della transizione italiana essa può essere considerata una spia, ma da dove questa transizione venga e dove stia andando può esserci detto solo dai modelli teorici entro i quali le misure vanno poste per determinarne (o relativizzarne) il significato e talvolta - vedremo - anche la corretta utilizzazione.

Il discorso ritorna così dove era cominciato, cioè alle predisposizioni meccaniche dei diversi tipi di sistema partitico elencate nella figura 1. Queste predisposizioni sintetizzano, in realtà, una sequenza causale più complessa, contenente cinque passaggi cruciali. Nel pluralismo moderato, la sequenza comincia dallo spazio ideologico «corto» indicato da tre a cinque partiti e, come tale, non contenente «apprezzabili segmenti di opinione estremista». I partiti, di conseguenza, formano poli «prossimi», cioè composti di attori che sottoscrivono la legittimità del

13 Bartolini (1995, 22-26). Sull'elevatissima volatilità preludente al crollo delle democrazie continentali fra le due guerre, e al passaggio dalla IV alla V Repubblica in Francia, vedi Bartolini e Mair (1990, appendice 2). Il rapporto inverso fra competitività ed efficienza economica è un altro indicatore di crisi sottolineato, fra gli altri, da Comiskey (1993). 
sistema e delle sue regole del gioco. In queste condizioni, «lo spazio è definito semplicemente dalle sue ali - sinistra e destra» e la competizione tende conformemente ad assumere una configurazione bipolare o, nel linguaggio della rational choice, è in equilibrio per due, e non più di due, schieramenti. Dati due soli poli, d'altro canto, la posta non può che essere l'elettorato intermedio fra loro, a sua volta disponibile, o fluttuante, perché non trattenuto (o non trattenibile stabilmente) da «terzi». E, a fortiori, la direzione della competizione sarà solo o prevalentemente centripeta, come già ricordato nella precedente discussione.

La situazione è speculare nel pluralismo polarizzato: come sappiamo, infatti, uno spazio «stirato» da più di cinque partiti segnala in questo caso un massimo di distanza ideologica fra le estreme, facendone due «poli opposti» includenti altrettanti partiti antisistema, o partiti «delegittimanti». Di conseguenza, «un terzo punto di riferimento - il punto centrale ... diventa non solo altamente visibile, ma a sua volta un polo, dotato di grande influenza» perché percepito come la «posizione sicura, cioè la posizione in grado di garantire al meglio la sopravvivenza di una data democrazia». In quanto tale, esso tiene «occupato fisicamente» l'elettorato moderato, il quale cessa così di essere «l'elettorato fluttuante per eccellenza e diventa, anzi, altamente stabile». Come dire che non solo il «sistema è ora tripolare o, eventualmente, multipolare»; ma poiché il voto moderato è occupato dal partito(i) di centro e intrasferibile alle estreme, la competizione tenderà forzatamente ad assumere un andamento centrifugo, piuttosto che centripeto (Sartori 1976, 347-351).

La direzione - centripeta o centrifuga - della competizione è, a sua volta, la variabile chiave, il «singolo elemento che di sicuro abbia decisive conseguenze per il rendimento complessivo di un sistema politico» (Idem, 293). Diversamente dalla competitività, cioè, essa è in grado di discriminare fra democrazie «facili e difficili», stabili e instabili, «efficienti» e «inefficenti» e, come tale, si potrebbe definire la condizione di funzionalità della competitività stessa. Se dunque discriminare si vuole, prima che quanta occorre chiedersi quale competizione (centrifuga o centripeta?). E poiché quest'ultima dipende - ho detto - da un'intera sequenza causale, la risposta va cercata ripercorrendola attentamente.

Per quanto ben nota, infatti, la sequenza è stata più spesso data per scontata che discussa o utilizzata in modo sistematico. 
E sono così rimasti nell'ombra - o nell'equivoco - non pochi suoi aspetti, di non poca rilevanza teorica ed empirica. Prendiamo, per cominciare, l'aspetto più esplicitamente sottolineato dal modello, cioè il rapporto fra il primo e l'ultimo anello della sequenza causale: estensione dello spazio ideologico e competizione. Senza dubbio, questo rapporto rappresenta il nocciolo del ragionamento che, se qualcosa spiega e predice, è che il «segreto della convergenza centripeta» o, viceversa, di una spinta centrifuga, sta nella «distanza lineare dell'ascissa», nella lunghezza del continuum sinistra-destra (Sartori 1976, 347). Ne consegue, piuttosto ovviamente, che la direzione della competizione è condizionata prima di tutto dall'andamento della polarizzazione, come rilevato da una varietà di indicatori: a livello di massa (sostegno al regime democratico, distribuzione delle autocollocazioni spaziali, distanza fra le autocollocazioni delle estreme); e a livello di élites (sistemi di credenze del personale politico, posizioni programmatiche e comportamenti parlamentari dei partiti, messaggi politici convogliati dai mass media).

$\mathrm{Su}$ questi indicatori, tuttavia, la letteratura recente è sorprendentemente muta, o almeno molto lacunosa. E mentre la lacuna può essere giustificata per le informazioni sulle élites, che sono di difficile raccolta, di ancor più difficile interpretazione e, comunque, non disponibili in forma sistematica, $i$ dati di massa ci sono e attendono solo un adeguato trattamento. Nel prossimo paragrafo, pertanto, dedicherò ad essi un ampio esame, che permetterà di aggiornare, con gli emendamenti e le estensioni opportuni, le ricerche di Sartori e Sani risalenti alla metà degli anni settanta. Le conclusioni - vedremo - suggeriscono una notevole evoluzione del sistema partitico italiano, che andrà così a colmare una prima disattenzione della letteratura sulla transizione. Ma di disattenzione ce n'è un'altra importante, riguardante il numero «appropriato» di poli da conteggiare in uno spazio «stirato» o «corto» e le corrispondenti opportunità, o incentivi, alla competizione centrifuga o centripeta presenti nel sistema. Nel modello che sto discutendo, la distinzione cruciale al riguardo è fra due o più di due; e, talvolta, essa è stata esplicitamente negata assumendo un universale «dualismo di tendenze» alla Duverger (Galli 1966); talaltra è stata semplicemente ignorata, attribuendo all'Italia una configurazione storicamente bipolare (Comiskey 1993, 379) ${ }^{14}$; più re-

14 Come dirò tra poco, lo stesso sembrano suggerire D'Alimonte e Bartolini. 
centemente, è stata equivocata, mescolando bipolarismo o multipolarismo con concentrazione/frammentazione del voto, ciò̀ con il formato bipartitico o multipartitico del sistema (Melchionda 1995, Vassallo 1995); e, infine, è stata posta in relazione - confusamente a dire il vero - con il sistema elettorale, da cui dipenderebbero il bipartitismo, il bipolarismo o, addirittura, la possibile restaurazione di una «egemonia neocentrista» (Melchionda 1995, 191; Chiaramonte 1995).

Che tutto questo porti solo fuori strada è stato più volte ribadito da Sartori (1982) e dovrebbe, comunque, risultare da quanto detto finora. Nondimeno, i fraintendimenti di Chiaramonte, Melchionda e Vassallo, in particolare, non sono solo il frutto di una lettura affrettata del modello, ma anche di irrisolte ambiguità inerenti alla sua applicazione nel contesto delle transizioni e, specificamente, di quella italiana. Data la loro natura dinamica, infatti, le transizioni presentano tipicamente fenomeni, eventi, caratteristiche in più o meno accelerato cambiamento, e il problema del numero dei poli non fa, ovviamente, eccezione. Con questo, resta fermo che, di regola e alla lunga, il bipolarismo interseca il numero dei partiti, cioè coesiste benissimo con il pluripartitismo, mentre è incompatibile con la polarizzazione, se e a misura che essa segnala uno spazio ideologico sufficientemente esteso da far posto ad un terzo polo. Ma, avendo a che fare con processi piuttosto che con stati, l'analista della transizione si troverà probabilmente davanti a «casi misti», combinazioni alternative a quelle normalmente spiegate e predette dal modello: per esempio, due poli polarizzati, ovvero tre (o più) poli in competizione su uno spazio ideologico «corto», o che va restringendosi (fig. 1, col. VI).

Di queste «misture dinamiche» o casi «in transito da una casella ad una altra», Sartori $(1976,287)$ osserva che sono un'ottima cosa, perché «documentano la sensibilità della tassonomia al cambiamento». Poiché però non aggiunge altro, si intende che la conferma della sensibilità è attesa a posteriori (cioè, a cambiamento completato), piuttosto che anticipata dall'analisi del cambiamento in corso. Questo, a sua volta, lascia i casi misti in quanto tali inesplorati e, quindi, il passaggio dal multipolarismo al bipolarismo (o viceversa) nella vaghezza. Ma la vaghezza può essere rimediata ricorrendo ad appositi indicatori di instabilità dei casi misti e rendendone così prevedibile il punto di arrivo.

Nella concreta situazione che ci interessa, gli indicatori do- 
vrebbero fornire informazioni sul destino del polo di centro allorché le stipulazioni dei modelli ne prevedano lo «schiacciamento» per convergenza delle ali su uno spazio competitivo «corto», ma - nelle more della transizione - esso non risulti ancora scomparso. Basta questa «resistenza» per parlare di persistente competizione tripolare o per accreditare la possibilità di un' «egemonia neocentrista», magari favorita da un dato sistema elettorale? Gli autori che lo sostengono - ho già detto - confondono multipolarismo con multipartitismo e, così facendo, sbagliano, ad un tempo, interpretazione del modello ed indicatori appropriati. Secondo il modello, infatti, uno o più partiti di centro fanno un polo non perché esistano o, eventualmente, resistano, ma in quanto «scoraggino la centralità», cioè le tendenze centripete del sistema politico». E la sequenza causale schematizzata più sopra suggerisce con chiarezza che queste tendenze sono scoraggiate e una «spinta centrifuga è impressa proprio dal centro metrico dello spettro» se, e solo se, l'elettorato moderato è «fisicamente occupato» e, come tale, sottratto alla competizione (Sartori 1976, 135, 349-350).

Nel corso di una transizione, per converso, sono indicatori di «liberazione» di voti precedentemente «occupati» che possono segnalare se, e fino a che punto, il numero dei poli e le associate meccaniche competitive stiano cambiando. E poiché ciò significa che l'attenzione deve cadere sull'instabilità (o mobilità, o volatilità) elettorale, sembrerebbe così di sfondare una porta aperta, dato che di questo si occupa, naturalmente, l'intera letteratura sul caso italiano. $\mathrm{Ma}$, se posta nel contesto della precedente discussione, anche un'ovvietà può diventare istruttiva, può segnalare qualcosa che è finora sfuggito ad altri autori. Come ho appena detto, infatti, nel passaggio dal pluralismo polarizzato al pluralismo moderato, l'aspetto cruciale è la mobilità del centro, la «liberazione» dell'elettorato moderato e il suo rientro nel mercato competitivo. Dunque, quando si esaminano i risultati elettorali con la classica misura della volatilità interblocco (o fra poli), non tutte le aggregazioni possibili sono da considerare equivalenti, come sembrano intendere D'Alimonte e Bartolini (1995, 446-449). Da un punto di vista teorico, l'aggregazione correttamente informativa è quella che coglie, appunto, lo svuotamento del terzo polo storico, della quale i due autori danno diverse versioni, più o meno plausibili e da discutere più avanti; ma non è plausibile, né corretto, affiancare a queste versioni il calcolo della volatilità sinistra/destra (447, tab. 
5) che, assorbendo il terzo polo in quest'ultima, ripete il doppio errore già ricordato: di interpretazione, in quanto viene così accreditata, perlomeno implicitamente, una configurazione bipolare del pluralismo polarizzato italiano; e, a fortiori, di verifica, perché togliere di mezzo i trasferimenti dal centro alle ali equivale a sopprimere in partenza la «prova» della transizione, o almeno a minimizzarla ${ }^{15}$.

Per di più, il rischio di minimizzazione è accentuato dal fatto che la volatilità aggregata si limita a segnalare gli spostamenti effettivi minimi di voti entro e/o fra i blocchi (Przeworski 1975, 53; Bartolini 1986, 27-34). Come tale, la misura «sottostima gli spostamenti individuali che, a loro volta, sottostimano la reale disponibilità dell'elettorato», ossia il potenziale di competizione nel sistema (Bartolini 1995, 11). Oltre che da riorientare teoricamente (sugli spostamenti dal centro), l'analisi della volatilità va dunque accompagnata da indicatori più inclusivi, se si vuole approssimare meglio la portata della transizione. Il più usuale di questi indicatori è rappresentato dai sondaggi sulle intenzioni di voto che, nonostante varie distorsioni, permettono stime normalmente superiori ai valori della volatilità aggregata (Biorcio e Natale 1989). Ma poiché anche queste stime sono approssimate per difetto (Mannheimer 1986; Corbetta et al. 1988), un altro strumento di controllo può essere suggerito, una volta di più, dal modello di Sartori.

Come si ricorderà, infatti, il modello àncora la forza del terzo polo, la sua capacità di «occupare» l'elettorato, all'esigenza di quest'ultimo di trovare una «posizione sicura», definita come la «posizione che garantisca la sopravvivenza della democrazia». Nel pluralismo polarizzato, cioè, stare al centro risponde a una «logica di difesa dalle estreme» e mantiene rilevanza sistemica in quanto il corrispondente posizionamento è sostenuto da questa motivazione. Con lo stesso metro, pertanto, il declino della motivazione appropriata fornirà un segnale di più di disponibilità (o di mobilità potenziale), specie se accompagnato da crescente insoddisfazione dell'elettorato moderato verso i partiti di

15 Naturalmente D'Alimonte e Bartolini $(1995,448)$ cercano di cautelarsi in anticipo da obiezioni sostenendo che l'aggregazione bipolare è ipotizzata per il futuro, non retrospettiva. Ma allora essa non può essere utilizzata per sottolineare la continuità con il passato, che è quanto i due autori intendono quando la commentano come prova di «un interscambio minimo tra blocchi e di una volatilità che si esprime quasi interamente all'interno del blocco di centro-destra». 
riferimento tradizionali. E poiché - vedremo - entrambi i processi sono documentati dai sondaggi, anch'essi vanno messi nel conto di un bilancio adeguato della transizione.

In altre parole, e concludendo, la transizione è un fenomeno altamente complesso, del quale la volatilità aggregata rappresenta un fattore, ma non certo il solo importante, né il più significativo teoricamente. Quando usata per conto proprio, anzi, essa si riduce a misurare (per difetto) una variabile (competitività) che, a sua volta, è fuorviante e può condurre nel vicolo cieco della post-dizione, se non inquadrata nel modello teorico che spiega e predice la logica del pluralismo polarizzato e del pluralismo moderato e le loro condizioni di mutamento. Appunto per questo, mi sono preso la pena di esaminare in dettaglio i due tipi, di ricostruirne con cura le stipulazioni in merito alla meccanica e, prima di tutto, al formato dei sistemi partitici. Resta ora da vederne l'applicazione al caso italiano, che è oggetto del prossimo paragrafo.

\section{Dal modello al caso}

Qual è il formato del sistema partitico italiano dopo la riforma elettorale e il voto critico del 1994? Per rispondere all'interrogativo, si possono usare gli indicatori quantitativi di rito (indice di frazionalizzazione, numero effettivo dei partiti, concentrazione del voto, numero di gruppi parlamentari), come fanno le analisi recenti di Morlino (1995) o di D'Alimonte e Bartolini (1995, 431-443). E con questo approccio è difficile non convenire che le nuove regole (semi)maggioritarie hanno avuto poco o punto effetto, che il sistema rimane un «caso di pluralismo estremo» $\mathrm{o}$, addirittura, appare più frammentato oggi che negli anni ottanta. D'altro canto, il limite di queste conclusioni è che esse prendono a valore facciale misure che 1) sommano tutte le unità del sistema (anche se non tutte ricevono lo stesso peso); e 2) trattano sistemi fortemente eterogenei (cioè, l'Italia prima e durante la transizione) in maniera continua, sottovalutandone così le irriducibili discontinuità ${ }^{16}$.

Come sappiamo, il primo limite può essere corretto facendo ricorso alle regole di conteggio di Sartori, che i tre autori peral-

16 L'incapacità di tener conto di discontinuità, ovviamente, è intrinseca agli indici matematici, per questo opportunamente criticati da Sartori $(1976,304-315)$. 
tro menzionano, seppur en passant. Ma le regole - ricordavo nel precedente paragrafo - presuppongono due condizioni di applicabilità per essere usate sensatamente, e queste condizioni sono cruciali appunto perché mettono in risalto, e permettono di tenere sotto controllo il secondo limite, cioè la discontinuità fra i sistemi in esame. Prendiamo, infatti, la condizione di gran lunga più importante, la quale circoscrive il calcolo della frammentazione (in termini di potenziale di coalizione e potenziale di intimidazione) ai partiti «veri», e ne esclude le mere «etichette», le «coalizioni sfilacciate di notabili cangianti da un'elezione all'altra». A questa stregua, partiti «veri» sono, per Sartori, i partiti strutturati e non c'è dubbio che, fino alla fine degli anni ottanta, tutte le forze politiche italiane lo fossero, in maggiore o minore misura. $\mathrm{Ma}$, con lo stesso metro, è altrettanto certo che la prima, vistosissima, discontinuità introdotta dalla transizione sia stata una destrutturazione di portata radicale, senza precedenti nazionali e comparati, dei partiti e dello stesso sistema partitico. Senza entrare nei dettagli del fenomeno ${ }^{17}$, questa situazione lascia dunque due possibilità di scelta: o porre l'Italia fra i sistemi atomizzati, cioè in «un punto al quale non è più necessario contare accuratamente» perché il «numero dei partiti - dieci, venti, o più che siano - fa poca differenza» (Sartori 1976, 125); o, più moderatamente, considerarla in stato di semiatomizzazione e distinguere conformemente le «etichette notabilari» propriamente dette dagli attori più «resistenti» alla transizione, o visibilmente in corso di ri-strutturazione.

Per molti motivi, la seconda scelta - la meno facile - è anche la più realistica: tutti i resoconti, infatti, confermano che alcuni partiti tradizionali hanno saputo contenere la propria crisi entro i limiti di un cambiamento graduale e controllato (PDS), o hanno solo beneficiato della crisi altrui elettoralmente e proceduto ad un adattamento significativo, ma nella continuità (AN); l'altro partito importante della sinistra - Rifondazione - si può dire «nato vecchio»o, se si vuole, già strutturato, in qualità di erede di risorse organizzative, personale ed elettorato ex-PCI; con varie attenuazioni, e fatto salvo il crollo elettorale, lo stesso vale per quegli ex-democristiani (Popolari di Bianco e, forse, CCD) che sono riusciti a conservare il tanto di apparato e zone di insediamento tradizionali risparmiati dalla transizione. Di continui-

17 Sul quale, vedi Morlino e Montero (1994), Morlino (1995) e Bardi (1996). 
tà, invece, non si può naturalmente parlare per partiti nuovi come Forza Italia e Lega Nord; ma anch'esse appaiono organizzazioni «vere», che hanno perlomeno superato la soglia della strutturazione in termini di una pluralità di indicatori ${ }^{18}$.

Secondo me, però, l'elenco finisce qui: è questo il confine oltre il quale comincia l'atomizzazione e non c'è più niente da contare, a parte una girandola di sigle in continuo cambiamento (Di Virgilio 1995). Queste sigle, infatti, sottintendono certo il grande agitarsi di una pletora di notabili, più o meno volubili e vocianti; ma dietro di loro non esistono strutture organizzative, «leggere» o «pesanti» che siano, se si eccettuano gli staff privati, o gli «amici», dell'uno o dell'altro personaggio; gli eletti, che sono invece numerosi, devono notoriamente il seggio alla «proporzionalizzazione» del maggioritario (cioè alle concessioni dei grandi partiti); e quanto ai loro (pochi) voti, l'andamento congiunturale è assai negativo $(-52 \%$ dalle politiche alle regionali 1995) e quello a lungo termine promette peggio, perché entrambi i fattori strutturali del pluralismo estremo (segmentazione culturale e polarizzazione) sono in declino, se non già virtualmente irrilevanti ${ }^{19}$.

A questo si potrebbe obiettare che, finché non scompaiono, partitini e notabili mantengono poteri di coalizione, o di ricatto, che non permettono comunque di scontarli. Ma l'argomento fraintende banalmente il significato delle due nozioni, che implicano - com'è ovvio - l'autonomia di chi esercita quei poteri. E senza voti sufficienti per avere seggi, l'autonomia si esprimerà nei comportamenti verbali o, al più, nella formazione dei gruppi parlamentari. Ma le decisioni chiave (cioè la scelta del governo da sostenere, o far cadere, e il voto legislativo) saranno sistematicamente allineate alle strategie dei grandi partiti. Tanto è vero, che la XII legislatura non offre un solo esempio di defezione degli epigoni del PSI, del PRI, del PSDI, del PLI, o altri «cespugli», mentre, quando questi ultimi erano davvero autono$\mathrm{mi}$, la sorte delle coalizioni dipendeva da loro almeno quanto dalla DC.

18 Oltre alla letteratura citata alla nota precedente, vedi Diamanti (1995); Ignazi (1995); Maraffi (1995).

19 Sulla «proporzionalizzazione» del maggioritario, vedi Di Virgilio (1995) e Bartolini e D'Alimonte (1995). I fattori del pluralismo estremo sono quelli che emergono dalla ricerca di Bartolini e Mair (1990, cap. 10). Sul declino della segmentazione in Italia, vedi, riassuntivamente, Cartocci (1994) e, sulla polarizzazione, più avanti. 
D'accordo con Sartori, dunque, ribadisco che gli pseudopartiti non contano, né vanno contati, e che questo ci lascia con sei attori rilevanti: AN, FI, PPI, Lega Nord, PDS, Rifondazione. Non solo. La qualificazione di uno di questi attori appare dubbia sotto il profilo dell'altra condizione di applicabilità delle regole di conteggio, quella che richiede l'allineamento dei partiti su una stessa ed unica dimensione di competizione, la dimensione sinistra-destra. Mentre questo requisito era certamente soddisfatto da tutti i soggetti del pluralismo polarizzato italiano, infatti, la seconda discontinuità introdotta dalla transizione è il peculiare posizionamento della Lega Nord, che - almeno nella più recente strategia della propria leadership - tende a rivendicare la sua unicità, a presentarsi come un «giocatore solitario», irriducibile a scendere sul terreno, e a condividere le regole altrui. Così facendo, la Lega rivendica il suo «dominio di identificazione» (cioè, il programma secessionista) e sembra volersi rinchiudere - fra molte contraddizioni, in verità - dentro di esso. Ma poiché non c'è nessuno (al momento $e$ in prospettiva) che la segua, la sua scelta «non aggiunge altre dimensioni di competizione ... e documenta, invece, una posizione fuori dalla corsa» $o$, se si vuole, una posizione sistemicamente irrilevante ${ }^{20}$.

Questa conclusione è avvalorata se si tiene conto che isolarsi nel «dominio di identificazione» comporta vantaggi solo a breve termine, mentre «con il tempo, il partito interessato dovrà affrontare il problema di tenere il suo seguito lontano dalla dimensione in cui la competizione ha luogo e finirà probabilmente per perderlo» (Sartori 1976, 354, n. 51). Conformemente, infatti, la Lega ha lasciato l'asse sinistra-destra quando si è resa conto di venire progressivamente schiacciata dai suoi competitori, ma solo per misurarsi con un crescendo di altre difficoltà: dalla neutralizzazione della questione federalista, ormai divenuta una valence issue priva di potere discriminante; al superamento della contrapposizione «vecchio-nuovo» che le aveva assicurato i primi successi; alla defezione di buona parte dei ceti medi imprenditoriali, sicuramente nient'affatto propensi alla «secessione»; al pessimo risultato delle regionali 1995, che lascia il partito al minimo storico nelle roccheforti tradizionali e al $5,5 \%$ del voto nazionale ${ }^{21}$.

20 Sartori $(1976,340)$.

21 Sulle difficoltà della Lega, mi sono limitato a riassumere l'analisi di Diamanti (1995, 127-174): Il dato elettorale è calcolato sui risultati ottenuti dalle liste regionali 
Indubbiamente, peraltro, isolamento e declino non tolgono che la Lega continui a controllare non pochi collegi nel Nord e in altri sia arbitro della contesa fra i maggiori schieramenti. Ma dovrebbe essere chiaro che, da un punto di vista teorico, questo non ne fa un attore più significativo di partiti con analogo potere elettorale, come il Front Nationale lepenista o i comunisti spagnoli. E anche la più ovvia prova di rilevanza (partecipazione ai governi Berlusconi e Dini) controbilancia solo in parte le precedenti osservazioni perché non è detto sia confermata, nel prossimo futuro, se il «secessionista» e «xenofobo» Bossi fa sul serio. Tenendo conto anche delle incertezze e delle ambiguità di una situazione in movimento, dunque, direi che il formato attuale del sistema - sei partiti - presenta un potenziale di riduzione a cinque, eventualmente potrebbe rimanere in bilico fra $\mathrm{i}$ due valori, ma quasi sicuramente non è destinato ad andare oltre.

Se la conclusione è ragionevole, la questione successiva è se, con il formato, si sia modificata la meccanica del sistema, e fino a che punto. Come ho argomentato dettagliatamente, per questo non basta esaminare i livelli di competitività, che portano fuori strada in assenza di preventive informazioni sulla polarizzazione, sul numero di poli e, attraverso di esse, sulla direzione della competizione. Prima di quei livelli, dunque, discuterò le variabili più «strutturali», a cominciare dalla polarizzazione e, precisamente, dal suo andamento a livello di massa. Gli indicatori pertinenti - ricordavo - sono (almeno) tre, e vale la pena di sottolineare che quello veramente cruciale è la misura della distanza ideologica, calcolata sulle autocollocazioni sinistra-destra. Nel modello di Sartori, però, la questione è, in verità, affrontata da una varietà di punti di vista o se si vuole, per approssimazioni successive. E il primo passo di questa strategia richiama l'attenzione sul fatto che uno «spazio dilatato» identifica società caratterizzate da «linee di divisione molto profonde», «basso consenso» e bassa legittimità del sistema politico democratico (Sartori 1976, 135 e 349). L'evoluzione di queste caratteristiche, a sua volta, può essere rilevata empiricamente dai dati di sondaggio sul sostegno proregime a livello di massa, sui quali Morlino e Montero (1994) e Morlino e Tarchi (1995) hanno dato di

della Lega, con l'aggiunta del voto riportato in Trentino e Friuli nel 1994. La percentuale, quindi, risulta dalla divisione per il totale dei voti validi alle elezioni politiche. 
recente articolati contributi. Senza entrare nei dettagli, le loro conclusioni possono essere riassunte in quattro punti, di evidente interesse in questa sede. Primo, la democrazia gode oggi in Italia della preferenza di una percentuale di elettori molto alta e non inferiore alla media comunitaria. Secondo, a questa ovvia prova di legittimità si accompagnano livelli storicamente forti e crescenti di insoddisfazione per il rendimento delle istituzioni, o, se si vuole, per l'efficacia decisionale, per il policy output, del sistema. Come tale, e come confermato da altri indicatori, l'insoddisfazione è di natura prevalentemente «pragmatica», cioè nutrita soprattutto da elettori moderati scontenti di come sono governati, ma non disposti a mettere in questione la stabilità del regime. Quarto, la sua esplosione con la transizione non segnala, dunque, crescente polarizzazione ma, al contrario, la caduta dei vincoli tradizionalmente posti da quest'ultima all'espressione di atteggiamenti critici (sul funzionamento dell'amministrazione pubblica, sulla gestione della crisi economica, ecc.) e a conformi cambiamenti delle scelte di voto.

Se questa interpretazione è corretta, insomma, l'Italia potrebbe essere ritenuta in rapido avvicinamento al modulo tipico delle democrazie legittime e consolidate: bassa (o decrescente) polarizzazione accompagnata $\mathrm{da}$ intensificate tendenze degli elettori ad utilizzare voice ed exit per sanzionare l'offerta programmatica dei partiti e il loro effettivo operato. Anche se a prima vista non sembra, il mix è tutt'altro che paradossale, poiché proprio un livello sufficientemente elevato di consenso di fondo assicura che l'insoddisfazione, la competizione, l'opposizione possano essere messe in atto a tutto campo senza rischio di delegittimare pericolosamente il sistema (Lijphart 1984, 2123). Tuttavia, prima di arrivare ad una conclusione di tanta portata, vale la pena di controllare gli altri due indicatori suggeriti dal modello, che insistono entrambi sulle autocollocazioni spaziali sul continuum sinistra-destra. Lasciando la variante più importante (misura della distanza) per ultima, qualche informazione utile si può cominciare a trarre dalla distribuzione di queste collocazioni. Da un punto di vista teorico, infatti, è evidente che diverse distribuzioni hanno conseguenze altrettanto diverse per le «tattiche remunerative di competizione elettorale» nel sistema: se esse contengono cospicue concentrazioni di autocollocazioni a sinistra e a destra, cioè, i partiti alle ali (e alle «mezze ali») saranno scoraggiati dal convergere al centro dalla preoccupazione di perdere più sostegno fra gli «estremisti» di quello 
guadagnato nell'opinione moderata ${ }^{22}$; mentre con ali sottopopolate il «ricatto» avrà poco o punto effetto, e la competizione downsiana (o centripeta) tenderà a prevalere. In base a queste premesse, Sartori (1982, 263-269) continuava a classificare l'Italia nel pluralismo polarizzato all'inizio degli anni ottanta, e poteva citare a sostegno le corrispondenti distribuzioni, documentate dalla ricerca otto Nazioni del 1974-76. Per controllarne l'andamento nel tempo, ho riportato queste distribuzioni nella tabella 1, insieme alle successive serie quinquennali disponibili.

Come si vede, la varietà di fonti e l'eterogeneità dei campioni non impediscono di rilevare una tendenza costante e marcata alla riduzione delle autocollocazioni a sinistra, passate nel 1995 a circa la metà del valore del 1975 . Per quanto riguarda le posizioni intermedie, invece, l'eterogeneità dei dati si fa sentire perché è probabilmente la causa principale delle forti fluttuazioni interclasse; ma il «disturbo» si riduce molto (e scompare se si confrontano direttamente 1975 e 1995) quando le tre classi vengono sommate e danno così un'opinione moderata complessivamente stabile, oscillante fra il 70 e l'80 per cento del totale. Di conseguenza, l'andamento che realmente lascia perplessi è l'impennata delle autocollocazioni di destra, troppo forte per essere ridotta ad una distorsione tecnica e in palese contrasto con la depolarizzazione a sinistra. Sembrerebbe infatti che la prima controbilanci la seconda e che la spinta centrifuga, a suo tempo rilevata da Sartori da una parte dello spettro si sia spostata dall'altra, continuando a suggerire uno «stato di estremizzazione» del sistema. E, certo, si potrebbe rispondere che, a questo effetto, contano soprattutto le mode e l'asimmetria della distribuzione (Sartori 1982, 264-265) e che, da entrambi i punti di vista, l'attuale struttura della curva italiana non è mai stata più simile a quelle (unimodali e simmetriche) della Gran Bretagna o della Germania. Tuttavia, il fatto che essa continui a differirne perché molto più popolata alle ali (e più schiacciata al centro) non può essere minimizzato e impone di farsi le domande veramente cruciali: quali ali? e quanto distanti?

A metà degli anni settanta Sartori $(1982,256-269)$ rispondeva identificando le ali nei due partiti antisistema (PCI e MSI) e calcolando fra loro la distanza di gran lunga maggiore nel suo

22 E, aggiunge Sartori (1976, cap. 10), anche i partiti di centro punteranno ad estendere il loro appello «a macchia d'olio» a destra e a sinistra, dando così il loro contributo alle spinte centrifughe del sistema. 
ТАВ. 1. Autocollocazioni sinistra-destra (\% su risposte valide)

\begin{tabular}{lccccc}
\hline & 1975 & 1980 & 1985 & 1990 & 1995 \\
\hline Sinistra & 24,3 & 18,9 & 17 & 13,9 & 13,4 \\
$\begin{array}{l}\text { Centro- } \\
\text { sinistra }\end{array}$ & 26,8 & 26,9 & 25,7 & 26,4 & 17,8 \\
Centro & 34,4 & 40,2 & 40,8 & 42,5 & 33,2 \\
$\begin{array}{l}\text { Centro- } \\
\text { destra }\end{array}$ & 9,5 & 9,6 & 11,3 & 10,8 & 20,4 \\
Destra & 5 & 4,4 & 5,1 & 5,3 & 15,1 \\
\hline
\end{tabular}

Fonte: 1975: elaborato da Sartori $(1982,258)$; 1980-1990: Eurobarometro; 1995 (3. 6 novembre): ISPO.

campione, e più che doppia rispetto a Gran Bretagna e Germania. Prendendoli di nuovo come termini di confronto, la tabella 2 aggiunge a quei dati tre rilevazioni più recenti, che documentano, apparentemente, una straordinaria vischiosità: distanze pressoché immutate, divari altrettanto stabili fra paesi e dunque - si direbbe - uno spazio italiano persistentemente dilatato, contenente partiti «alienati, se non nemici» (RC e AN?), i quali «non desiderano, né hanno molto da guadagnare dalla competizione centripeta», poiché i loro obiettivi sono serviti al meglio dalla «distruzione del sistema», cioè «da più, non meno, polarizzazione» (Sartori 1976, 350).

Naturalmente, nessuno, a cominciare da Sartori, sottoscriverebbe oggi una conclusione del genere; e, di conseguenza, si sarebbe tentati di dubitare, certo al presente, ma forse anche retrospettivamente, dei meriti di una «variabile» che si comporta piuttosto come una costante, cioè che è poco o punto sensibile a cambiamenti della portata di una transizione di regime. $\mathrm{Ma}$ un'altra possibilità è che l'insensibilità sia l'artefatto di un'errata base di calcolo della misura o, se si vuole, del suo mancato adeguamento alla mutata situazione.

L'osservazione è suggerita dal fatto che, nel modello sartoriano, i soggetti della meccanica del sistema sono i poli, non i partiti in quanto tali. Correttamente, pertanto, la distanza veniva misurata in passato dalle differenze PCI-MSI: nel pluralismo polarizzato italiano, partiti e poli laterali coincidevano ad ogni effetto. $\mathrm{Ma}$, secondo me, è difficile sostenere altrettanto dopo 
TAB. 2. Polarizzazione sinistra-destra: distanze fra partiti e distanze fra poli

\begin{tabular}{lcccll}
\hline & 1975 & 1983 & 1993 & & 1995 \\
\hline Italia & & & & RC & 2,7 \\
PCI/PDS & & & & PDS & 3 \\
PSI & 2,5 & 2,5 & 2,4 & Lega Nord & 5,5 \\
PSDI/PRI & 3,7 & 3,5 & 3,6 & PPI & 5,7 \\
DC & 4,8 & 4,9 & - & FI & 6,9 \\
PLI & 5,9 & 5,7 & 5,7 & AN & 8,1 \\
MSI & 6,5 & 6,8 & - & & 8,1 \\
Distanza massima & 8,3 & 7,5 & 7,9 & & \\
& 5,8 & 5 & 5,5 & & \\
Gran Bretagna & & & & & \\
& & & & & \\
Laburisti & 4,4 & 4,4 & 4 & & \\
Liberali & 5,6 & 5,3 & 5 & & \\
Conservatori & 7,2 & 7 & 7 & & \\
Distanza massima & 2,8 & 2,6 & 3 & & \\
Germania & & & & & \\
& & & & & \\
Verdi & & & & & \\
SPD & - & 3,6 & 4,3 & & \\
FDP & 4,5 & 4,5 & 4,5 & & \\
CDU & 5,8 & 5,9 & 5,4 & & \\
Distanza massima & 7 & 6,7 & 6,8 & & \\
\hline
\end{tabular}

* Distanza fra i poli di centro-sinistra (posizione media di RC + PDS + PPI $=3,3$ ) e centro-destra (posizione media di AN + FI + CCD = 7,5).

Fonte: 1975: Sartori (1982, 257-258); 1983 e 1993: Gunther e Montero (1994, tab. 12); 1995 (3-6 novembre): ISPO.

l'inizio della transizione e, specialmente, dopo le elezioni del 1994. Con tutta evidenza, infatti, transizione e sistema maggioritario hanno spostato il centro di gravità della competizione (elettorale ed interelettorale) dai partiti ai due blocchi di centro-destra e di centro-sinistra. E sebbene la coesione di questi blocchi (o poli) sia ancora discutibile e discussa, non c'è dubbio che essa abbia fatto tormentati ma rapidi progressi e che, dalle regionali del 1995, la loro rilevanza sistemica, sia decisamente prevalsa sugli scenari alternativi che D'Alimonte e Bartolini $(1995,460-466)$ potevano ancora prendere in considerazione subito dopo le consultazioni politiche ${ }^{23}$. A questa stregua, il cal-

23 Per usare il linguaggio dei due autori, infatti, il «centro ha scelto», anche se non unitariamente come essi avevano supposto. 
colo tradizionale riportato nella tabella 2 (primo valore della colonna 4) non offre più un termine di confronto intranazionale ed internazionale omogeneo. Per continuare a misurare lo stesso fenomeno nella nuova situazione, infatti, bisogna spostare l'attenzione sul secondo valore della colonna, che calcola le distanze fra gli attuali poli e, poiché questi constano di diversi partiti, le esprime tenendo conto della loro forza relativa ${ }^{24}$. Il risultato, come si vede, è un cambiamento finalmente apprezzabile: dal 5,8 del 1975 al 4,2 del 1995, la diminuzione della distanza è dell'ordine del $28 \%$; di conseguenza, il divario con Germania e Gran Bretagna, seppur persistente, va ai minimi storici; e questo si riflette in un «indice di polarizzazione» italiano disceso a 0,47 (contro lo 0,64 del 1975), mentre quelli degli altri due paesi sono rimasti immutati a 0,28 e 0,33 rispettivamente (Sartori 1982,261 ).

Se il mio ricalcolo è giustificato, dunque, concluderei che la depolarizzazione è in corso e, semmai, se ne può discutere l'entità, non l'esistenza. Per essere fruttuosa, però, la discussione va condotta il più esaurientemente possibile, utilizzando cioè quanti altri indicatori forniscano informazioni appropriate. Al processo di depolarizzazione ideologica, per esempio, si accompagna di sicuro la virtuale scomparsa della polarizzazione sulla dimensione religiosa, che nel 1975 quasi raddoppiava le distanze complessive fra i poli ${ }^{25}$. E sebbene la distanza ideologica sia a rigore sufficiente per spiegare la logica competitiva del sistema, va da sé che la caduta delle identificazioni confessionali ha rappresentato, e rappresenta, un incentivo chiave alla conver-

24 Il calcolo è stato effettuato sommando i valori complessivi delle autocollocazioni degli elettori dei partiti assegnati a ciascun polo e dividendo le somme ottenute per il numero totale di elettori di questi partiti. Una misura analoga è usata da Bartolini e Mair $(1990,204)$ per calcolare le distanze programmatiche fra blocchi nella loro ricerca sulla volatilità. Tuttavia, mentre essi effettuano la ponderazione sui risultati elettorali dei partiti, ho preferito in questa sede utilizzare i dati di sondaggio, che danno per superati, e non di poco, i rapporti di forza intrablocco usciti dalle elezioni del 1994. Un'altra differenza è che Bartolini e Mair includono nell'uno o nell'altro blocco tutti i partiti, mentre nel presente calcolo sono considerati solo quelli rilevanti, esclusa la Lega, che, al momento, non sembra disposta a schierarsi. Il blocco di centro-sinistra, dunque, è composto da Rifondazione, PDS e PPI, e quello di centro-destra da An e FI, ai quali ho aggiunto il $\mathrm{CCD}$, che - si ricorderà - non era incluso nel mio calcolo del formato, ma appare, fra i partiti minori, l'unico sulla soglia dell'autonomia elettorale e, quindi, della rilevanza. Comunque sia, esso sposta la posizione media del centro-destra di una frazione insignificante $(0,1)$.

25 Sartori (1982, 273-279). Sul processo di secolarizzazione, vedi Cartocci (1994). 
genza centripeta, rendendo disponibile la stragrande maggioranza dell'elettorato (ex-cattolico) di centro agli appelli delle ali laiche. Foss'anche solo per questo, si deve dissentire dalla conclusione che «invece di essere il luogo geometrico dell'elettorato mediano e indeciso, il centro rimane occupato da un partito politico che il sistema maggioritario vorrebbe sfrattare ma che, in virtù della sua identità, del suo radicamento sociale e del legame con la Chiesa, non ha intenzione di sgombrare» (D'Alimonte e Bartolini 1995, 461). Nel frattempo, infatti, quel partito ha effettivamente «sgombrato», spaccandosi e rifluendo dall'una o dall'altra parte; e anche se è facile giudicare col senno di poi, sarebbe bastato - secondo me - guardare agli usuali indicatori di secolarizzazione per anticipare che, nell'Italia degli anni novanta, non c'è più posto per un polo confessionale (Cartocci 1994, 186-190). Ma è altrettanto certo che non c'è posto per un terzo polo laico, o, eventualmente, misto?

Come ho a lungo argomentato, la risposta dipende, appunto, dall'«occupazione» stabile, o dall'occupabilità, del voto moderato, e D'Alimonte e Bartolini, a quanto pare, sono d'accordo. Conformemente alla precedente citazione, però, la loro analisi tende a minimizzare la mobilità elettorale e, soprattutto, la mobilità verso sinistra. Per questo, essi cominciano con il fornire varie misure di volatilità interblocco, calcolate per aggregazioni che lasciano talvolta perplessi. Ho già ricordato, infatti, che se il problema è cogliere la transizione da un sistema tripolare ad uno bipolare non si può ragionare sui trasferimenti fra due soli blocchi (sinistra e destra), che gli autori presentano nella V colonna della loro tabella 5. Così facendo, si tratta l'Italia né più né meno come la Gran Bretagna e, come in Gran Bretagna, si riscontra un «interscambio minimo» che è, però, un mero artefatto operazionale della scelta di accorpare il centro alla destra, cioè di ridurre a movimenti interni i relativi trasferimenti. Se questa neutralizzazione ex ante della prova della transizione è - come credo - in contrasto con la teoria, la storia e il buon senso, altrettanto discutibili appaiono i calcoli delle due precedenti colonne: nella IV, il problema principale è l'invenzione di un blocco («nuovo centro-destra») che sottrae le perdite di PSI, PSDI, PRI e PLI dai guadagni di Radicali, FI e AN; nella III, invece, Radicali, Lega e FI compensano, entro lo stesso «grande centro», per DC e laici minori; cosicché, se è vero che viene ora abbandonata la configurazione bipolare, anche in queste varianti la distorsione rimane sensibile, perché esse con- 
ТАВ. 3. Volatilità totale, intra e interblocco, 1992-1994, 1992-1995*

\begin{tabular}{|c|c|c|c|}
\hline & 1992-1994 & & $1992-1995$ \\
\hline Volatilità totale & 39,4 & & 61,5 \\
\hline $\begin{array}{l}\text { Blocco nuova } \\
\text { sinistra }\end{array}$ & $+7,9$ & $\begin{array}{l}\text { Blocco nuovo } \\
\text { centro-sinistra }\end{array}$ & +19 \\
\hline $\begin{array}{l}\text { Blocco centro } \\
\text { pentapartito }\end{array}$ & -37 & $\begin{array}{l}\text { Blocco centro } \\
\text { pentapartito }\end{array}$ & $-53,3$ \\
\hline $\begin{array}{l}\text { Blocco nuova } \\
\text { destra }\end{array}$ & $+31,2$ & $\begin{array}{l}\text { Blocco nuovo } \\
\text { centro-destra }\end{array}$ & $+35,2$ \\
\hline Altri & $-2,9$ & Altri & $-4,6$ \\
\hline Volatilità interblocco & 38,1 & & 56 \\
\hline Volatilità intrablocco & 1,3 & & 5,5 \\
\hline
\end{tabular}

* Nuova sinistra: RC, PDS, Verdi, Neo-PSI, AD, Rete. Nuovo centro-sinistra: oltre ai precedenti, comprende Popolari, Patto Democratici e le altre liste circoscrizionali minori presentatesi nel comparto proporzionale nel 1995 e alleate nei listoni regionali progressisti. Nuova destra: FI, AN, Lega nord, Lista Pannella; Nuovo centro-destra: Forza Italia-Polo popolare, CCD, AN. Altri: 1994, liste minori; 1995, liste minori, Lega Nord, Pannella-Riformatori.

tinuano a confondere pezzi del vecchio terzo polo con pezzi dei nuovi, cioè fanno passare almeno una quota di volatilità interblocco per trasferimenti intrablocco.

Poiché nelle due varianti la distorsione è solo parziale, la volatilità interblocco che esse registrano cresce notevolmente rispetto ai livelli minimi della configurazione bipolare; ma poiché rimane una frazione pur sempre minoritaria $(40 \%)$ di quella totale, D'Alimonte e Bartolini $(1995,449)$ possono concludere che è perlomeno prematuro parlare di «un elettorato ormai svincolato dalle identità del passato e libero di scegliere in base all'offerta di mercato». Senonché, questo è proprio quanto suggerisce l'unica delle loro aggregazioni che - secondo me - sia in regola con le stipulazioni del modello sartoriano e che ho riportato nella prima colonna della tabella ${ }^{26}$. Essa, infatti, distingue correttamente ciò che caratterizzava il pluralismo polarizzato, ossia il vecchio polo prosistema («centro pentapartito»); inoltre,

26 Un'altra aggregazione ancora suggerita dai due autori è a sua volta discutibile perché attribuisce indebitamente le perdite del PSI storico alla sinistra, distorcendone così al ribasso la competitività. 
lascia dall'una e dall'altra parte i suoi competitori storici, più le forze nuove che, con loro, hanno concorso alla cattura dell'elettorato moderato nel 1994; e la «risposta» di quest'ultimo, dunque, è correttamente approssimata dall'elevatissima volatilità corrispondente $(38,1 \%)$, che rivela quanto poco rimanga del centro «occupato» del passato.

D'altro canto - si potrebbe obiettare - questa è, appunto, l'«istantanea» del 1994 e, come tale, non tiene conto degli sviluppi politici ed elettorali successivi, cioè della defezione della Lega Nord dal blocco di centro-destra e dei risultati delle regionali 1995. Ma il punto è se i due sviluppi siano in contrasto con il riallineamento bipolare del sistema, che è la tendenza strutturale evidenziata dalle elezioni politiche. E a me sembra piuttosto che essi la accentuino perché, come ricordato, i Popolari si sono nel frattempo schierati (a destra o a sinistra), mentre la Lega tentenna fra due comportamenti (l'autoisolamento scelto alle regionali e rapporti assai conflittuali con il centro-sinistra) che la rendono, in verità, inclassificabile. Se dunque si ridistribuiscono conformemente gli ex-democristiani e si sposta l'inclassificabile Lega fra gli «altri», l'aggiornamento dell'istantanea al 1995 dà i risultati della seconda colonna della tabella 3 che, riassuntivamente, commenterei così: al centro, ci sono certo molti elettori, come risulta dalle autocollocazioni; ma sono del tutto scomparsi i partiti e, a fortiori, il terzo polo; pertanto, ai moderati non resta che optare fra i poli rimanenti e a questi non resta che contendersene il sostegno convergendo verso di loro; la convergenza, a sua volta, può non esser perfetta, come attestato dalla riduzione ancora in corso della distanza ideologica fra centro-sinistra e centro-destra; ma a giudicare dalla volatilità interblocco 1994 e dal suo balzo al 56\% nel 1995, la riduzione si direbbe sempre più sufficiente per convincere la stragrande maggioranza degli elettori mediani che non ci sono rischi per la democrazia dall'una o dall'altra parte e che, dunque, è finalmente possibile scegliere fra le due.

Queste conclusioni - ritengo - sarebbero anche rafforzate se possedessimo misure di disponibilità più inclusive della volatilità, come quella proposta da van der Eijk e Oppenhuis (1991) per cogliere l'effetto combinato degli spostamenti effettivi e della propensione a spostarsi rilevata dai sondaggi. In attesa di dati più appropriati, però, basta un'occhiata ai sondaggi puri e semplici per rendersi conto che, dal 1993 almeno, le intenzioni di voto registrano fortissime oscillazioni; a loro volta, queste si tra- 
ducono in frequenti ribaltamenti di ipotetiche maggioranze; e l'unico trend estraibile dall'enorme incertezza è la rapida, continua ed apparentemente inarrestabile emarginazione degli ex-popolari e degli altri aspiranti a ricostituire il cosiddetto «grande centro ${ }^{27}$. Se dunque confrontassimo la struttura italiana delle intenzioni di voto con quella di altri paesi europei, non la troveremmo più «anormale» come in passato, cioè stabile e tripolare, $\mathrm{ma}$, al massimo, più dispersa, per effetto di una frammentazione peraltro irrealistica o, se si vuole, sistemicamente irrilevante. E se volessimo cogliere le motivazioni sottostanti a tale mutamento, ci troveremmo di fronte ad altre tendenze reciprocamente rinforzantesi: una forte caduta della «paura delle estreme», che nel pluralismo polarizzato teneva insieme i moderati in nome della «logica di difesa» della democrazia incarnata dal polo di centro; un'ancora più rapida ascesa della loro insoddisfazione verso di esso e i suoi eredi, evidentemente correlata al venir meno dei suoi punti di forza, o «meriti» storici; e la «liberazione» di una fortissima domanda di efficacia decisionale, non più compressa dalla tolleranza dell'immobilismo faute de mieux che un tempo rappresentava il rovescio della medaglia della rendita di posizione della DC e dei suoi alleati ${ }^{28}$.

Senza esagerare l'importanza di queste informazioni, sembra chiaro che aggiungerle al mio bilancio non fa che completarlo. $\mathrm{Ma}$ anche questo lascia in sospeso un problema, anzi il vero problema sollevato dall'analisi di D'Alimonte e Bartolini. Essi, infatti, potrebbero concedere - e credo concederebbero tanto più facilmente dopo il crollo e il riallineamento dei popolari che il bipolarismo è ormai dominante e l'elettorato mediano sempre più mobile. Ma la mobilità - continuerebbero ad obiettare - rimane «imperfetta», grande a destra e molto più ridotta a sinistra, dove permane un «divieto di svolta» che riflette «residui di polarizzazione», mantiene bassa la competitività del sistema $\mathrm{e}$, in definitiva, rende improbabile l'alternanza, cioè la prova decisiva che la democrazia italiana si è normalizzata, o si va normalizzando ${ }^{29}$.

Come si ricorderà, una possibile critica di questa imposta-

27 Faccio riferimento ai sondaggi presentati da Ricolfi $(1995,276)$ e al panel dell'ISPO supervisionato da Mannheimer. (1995).

28 I dati Eurobarometro sui quali si basano queste informazioni sono in Morlino

29 Bartolini e D'Alimonte (1995, 357-369). 
zione è stata più volte ribadita. Da un punto di vista teorico, erigere la competitività a criterio di classificazione e fare dell'alternanza il suo banco di prova significa confondere l'explanandum con l'explanans e, di conseguenza, abbandonare la spiegazione e la predizione per accontentarsi di post-dire sulla base dei risultati elettorali. D'altro canto, è anche vero che la posizione di D'Alimonte e Bartolini trae sostegno da alcuni dati empirici, che il rigore teorico non basta per cancellare. Vale la pena, quindi, di controllare questi dati, per accertare se essi sono veramente in linea con il «divieto di svolta a sinistra». Il primo dato concerne i collegi uninominali marginali, che vengono trovati pochi, soprattutto alla Camera, e assai disegualmente distribuiti sul territorio nazionale (Bartolini e D'Alimonte 1995, 327-340). Questo - osservano i due autori (1995, 454) - comporta «una bassissima competitività in termini di simmetria di voti. Le cifre nazionali sono, da questo punto di vista, illusorie perché nascondono le abissali differenze che, nonostante la strutturazione bipolare, nella maggior parte dei collegi, hanno separato i primi due candidati. Al Nord, dei 180 seggi della $\mathrm{Ca}$ mera ben 161 sono andati ai candidati del Polo della Libertà con uno scarto medio del $25 \%$ dei voti rispetto al candidato arrivato secondo ... Al Senato, i distacchi sono stati inferiori, ma pur sempre ragguardevoli ... La stessa situazione, rovesciata, si è verificata al Centro ... Solo il Sud ci ha fornito una mappa politica più variegata, evitando che in intere regioni o anche aree geografiche tutti o quasi i seggi fossero appannaggio di un'unica formazione o alleanza politica». E proprio grazie alla maggior competitività nel Sud «la sinistra ha ottenuto relativamente molti seggi ed ha evitato così una vera e propria disfatta» (Bartolini e D'Alimonte 1995, 371); ma poiché né il Sud, né le fortezze PDS del centro bastano a controbilanciare l'egemonia della destra nel Nord, nel suo probabile futuro ci sono poche chances di conquistare le quote di elettori moderati che sarebbero indispensabili per capovolgere il risultato del 1994.

Quanto è plausibile questa conclusione? Fondamentalmente, dipende dalle scelte operazionali compiute per determinare il numero di collegi marginali e, in secondo luogo, dai criteri eventualmente usati per decidere se essi sono «molti», «pochi» o «abbastanza». Quanto all'operazionalizzazione, però, trovo molto convincente l'obiezione di Melchionda (1995, 160) contro la misura (differenza semplice fra primo e secondo arrivato) con cui Bartolini e D'Alimonte distinguono fra collegi più o 
meno competitivi; seguirei, quindi, il suo suggerimento di utilizzare la differenza quadratica, che permette di tener conto «dei rapporti di forza relativi fra gli attori», inclusi i terzi; e, ancora d'accordo, concluderei che i collegi marginali diventano così poco meno della metà alla Camera e oltre la metà al Senato, contro il 29 e il 38,8\% calcolati da Bartolini e D'Alimonte ${ }^{30}$.

Cambiando misura, insomma, cambiano - di tanto, pare la competitività e l'apertura del mercato elettorale e si riducono, seppure più moderatamente, anche alcuni squilibri territoriali nella distribuzione dei collegi (Melchionda 1995, 163-167). $\mathrm{Ma}$ quale che sia la misura, ricordavo che occorre comunque un criterio per decidere se essa identifica «molti» o «pochi» seggi marginali. E poiché Bartolini e D'Alimonte $(1995,338)$ dichiarano esplicitamente che il criterio dovrebbe essere fornito dal «controllo comparato», vale la pena di osservare che esso non conferma le loro aspettative. In Gran Bretagna, infatti, i seggi marginali erano pari al 25,2\% nel 1992; durante il dopoguerra (1955-1992), la percentuale media è stata identica, con una punta massima del $31,6 \%$ e un minimo del 20,6\%; poiché sono calcolati su differenze fra primo e secondo fino al $9,9 \%$, questi valori sono per di più sicuramente superiori a quelli che si otterrebbero con il limite scelto da Bartolini e D'Alimonte $(8 \%)$; ma anche lasciando perdere la precisione, è chiaro che $\mathrm{i}$ marginali inglesi sono al di sotto del $29 \%$ della Camera e del $38,8 \%$ del Senato, per non parlare del 45 e del $55 \%$ calcolati da Melchionda ${ }^{31}$.

Con questo, non voglio certo dire che le elezioni del 1994 abbiano introdotto in Italia il «modello Westminster» e i suoi standard «virtuosi». Allo scopo, contare i collegi marginali non basta, ci vorrebbero perlomeno gli indicatori complementari suggeriti da Norris e Crewe (1994) e, comunque, molto altro ancora. Pertanto, mi limito a ripetere che i numeri citati provano semplicemente un livello di competitività sistemica, cioè un'apertura ed un'incertezza della corsa elettorale, di cui si possono discutere le precise dimensioni ed implicazioni, senza però negare che esso è comparativamente elevato, non basso, o, tantomeno «bassissimo». $\mathrm{Ma}$, detto questo, resta un ultimo argomento, anch'esso dovuto a D'Alimonte e Bartolini. 
Questo argomento - in verità, il più importante - addita la maggior debolezza della sinistra nell'eterogeneità della sua base, la quale include elettori moderati accanto ai postcomunisti di Rifondazione, ossia gruppi ideologicamente ostili, fra i quali permangono «residui di polarizzazione» che impediscono di sommare tutti i loro voti. Infatti - sostengono i due autori - «i candidati del cartello di sinistra vanno sistematicamente peggio delle liste progressiste nel loro complesso ... In alcuni casi, lo scostamento è minimo ... ma in altri è sensibile, visto che $\mathrm{i}$ candidati di RC perdono mediamente il $2,8 \%$ dei voti che invece raccolgono le liste di cartello ... Da dove vengono questi voti persi? La nostra ipotesi è che siano elettori ideologici, elettori di una sinistra moderata, che trovandosi nel proprio collegio un candidato di RC si rifiutano semplicemente di votarlo. Ma la stessa ipotesi possiamo applicarla al caso dei candidati di AD che pure non riescono a mobilitare tutto l'elettorato del cartello progressista ... In questo, trova conferma ... il permanere, pur con un grande elettorato mobile, di una forte cesura tra sinistra e centro-destra» che, ancora una volta, avvalora la scarsa competitività del sistema (Bartolini e D'Alimonte 1995, 364-367; D'Alimonte e Bartolini 1995, 455-457).

Come si vede, l'interpretazione insiste su un fattore strutturale (polarizzazione ideologica) dagli effetti tendenzialmente uniformi e di lungo periodo. A rigore, dunque, la sua persuasività andrebbe valutata alla distanza, cioè su una serie di conferme dei dati del 1994 eventualmente offerte dalle elezioni politiche successive. Solo così sarà possibile accertare se lo scarto sistematico negativo della sinistra (e l'altrettanto sistematico scarto positivo per la destra) non sia effetto (almeno in parte) di condizioni contingenti, come la diversa capacità dei due schieramenti di dare soddisfazione alla domanda di «facce nuove», a quanto pare fortemente espressa dall'elettorato nella passata consultazione $\mathrm{e}^{32}$. $\mathrm{Ma}$, a prescindere da questa alternativa, un confronto (benché non proprio omogeneo) è stato di recente proposto da D'Alimonte sui dati delle regionali che mostrano per sua stessa ammissione - una situazione in movimento. L'ammissione - beninteso - è cautissima e non toglie che a sinistra sia confermato «un risultato maggioritario negativo», sep-

32 Alle sistematiche differenze di sommabilità fra sinistra e destra, infatti, corrisponde un altrettanto sistematico minor ricambio dei candidati offerti dai progressisti rispetto a quelli del polo (Massari 1995; Mattina 1995). 
TAB. 4. Elezioni regionali 1995. Differenza tra voti di cartello e voti di lista in \% del voto di lista totale

\begin{tabular}{lcc}
\hline & Centro-sinistra & Centro-destra \\
\hline Piemonte & $+6,5$ & $+7,2$ \\
Lombardia & $-0,2$ & $+2,5$ \\
Veneto & $+1,4$ & $+5,8$ \\
Liguria & $+2,4$ & $+3,4$ \\
Emilia-Romagna & $+1,7$ & +4 \\
Toscana & $+2,8$ & $+5,2$ \\
Umbria & $+1,3$ & $+5,1$ \\
Marche & $+3,8$ & $+5,5$ \\
Lazio & $+7,8$ & $+8,6$ \\
Abruzzo & $+1,4$ & $+6,8$ \\
Molise & $+5,7$ & $+2,4$ \\
Campania & $+3,9$ & $+5,9$ \\
Puglia & $+1,5$ & $+7,3$ \\
Basilicata & - & +5 \\
Calabria & $+4,6$ & $+4,3$ \\
\hline
\end{tabular}

pur «complessivamente inferiore a quello delle politiche» $(1995,553)$. Per la sua dimostrazione, però, D'Alimonte si serve della differenza fra percentuali ottenute dai diversi valori assoluti dei comparti maggioritario e proporzionale, mentre, se si ripete il calcolo effettuato con Bartolini per le politiche ${ }^{33}$, il rendimento elettorale di entrambi i cartelli risulta sistematicamente positivo (tab. 4).

La ragione della discrepanza, naturalmente, è banale, cioè discende dalla forte eccedenza (oltre tre milioni di voti) registrata dai «listoni» regionali rispetto alle liste circoscrizionali. $\mathrm{Ma}$ meno scontato è che questo fatto introduce una specifica condizione di ammissibilità del calcolo di D'Alimonte e, soprattutto, modifica la sua rilevanza ai fini della valutazione della competitività della sinistra.

Per quanto riguarda l'ammissibilità, è chiaro che il nuovo calcolo proposto ha senso se si postula l'equidistribuzione fra gli schieramenti dei voti validi maggioritari in eccesso sulla proporzionale. Solo così è possibile considerare cruciale il fatto che «l'aumento dei voti validi ... non è stato neutrale rispetto alla forza relativa dei partiti», registrare quindi un incremento meno che proporzionale dei «listoni» del centro-sinistra e attribuirlo

33 Tale calcolo determina la differenza in valore assoluto fra voti di lista e voti maggioritari per ciascun schieramento e la esprime in percentuale del voto di lista totale. 
all'usuale spiegazione in termini di «voto ideologico», «residui di polarizzazione» e limitata sommabilità del suo elettorato (D'Alimonte 1995, 549-551).

Questa conclusione, in altre parole, è ora subordinata strettamente ad un assunto non richiesto per i dati delle politiche, nelle quali lo scarto fra le due arene elettorali era trascurabile; e, secondo me, ciò non è indifferente per la sua solidità, in quanto la postulata neutralità della distribuzione suscita almeno due obiezioni. La prima è suggerita dall'esplicita e pubblica delegittimazione del voto (solo) maggioritario da tempo proveniente, per ovvie ragioni di bottega, dai «cespugli» del centrosinistra e, soprattutto, da Rifondazione e dai neoalleati Popolari. Poiché niente di paragonabile avviene nel centro-destra, infatti, appare problematico ipotizzare una struttura di preferenze omogenea nei due elettorati o, se si vuole, la stessa propensione a votare per i cartelli e per le liste. Ma se la propensione ragionevolmente differisce, altrettanto ragionevolmente ci si dovrebbero aspettare rapporti maggioritario/proporzionale diversi; la differenza, a sua volta, andrebbe spiegata anche (se non solo) con i diversi messaggi convogliati sull'elettorato dei due campi; e questi messaggi, infine, non sarebbero da ricondurre tanto all'eterogeneità ideologica (che ne sarebbe, al più, una componente), quanto al differenziale di frammentazione che naturalmente incentiva i molti «piccoli» del centro-sinistra e i pochi «grandi» del centro-destra a porre l'enfasi sulle arene rispettivamente più convenienti.

A ciò la seconda obiezione aggiunge un elemento ancora più tangibile, che salta agli occhi se si guarda alle percentuali di voto proporzionale dei due schieramenti. In undici regioni su quindici il centro-sinistra sta sopra il $40 \%$ e in sei casi oltre il 50; il centro-destra, viceversa, è attestato fra il 30 e il $40 \%$ in sette casi, supera questa percentuale negli altri otto, ma solo in uno va poco oltre il 50 . Ci sono dunque differenze importanti e diffuse fra le soglie alle quali l'una e l'altra coalizione avrebbero dovuto aggiungere il sovrappiù di voto maggioritario; queste differenze, a loro volta, suggeriscono una difficoltà di crescita tanto maggiore, quanto più forte la posizione di partenza ${ }^{34}$; e poiché più forte è appunto la posizione tipica del centro-sinistra, il postulato di

34 Questa difficoltà può essere dovuta a vari motivi, ma il più importante sembra essere l'impulso smobilitante che gli elettori dello schieramento più forte ricevono dall'aspettativa di non poter aggiungere niente a una vittoria già scontata e completa. 
distribuzione neutrale ritorna discutibile e il suo più modesto rendimento rispetto al centro-destra diventa leggibile come «effetto soglia», piuttosto che come «effetto polarizzazione».

Come che sia, il vero problema è quanto rilevante è oggi il ragionamento discusso, cioè che cosa dimostrano di nuovo $\mathrm{i}$ dati delle regionali riguardo alla competitività del centro-sinistra. E, in proposito, anche D'Alimonte $(1995,22-23)$ non può evitare di ammettere una «differenza importante», perché «mentre per il 1994 si può effettivamente parlare di un centrosinistra che «perde pezzi» tra proporzionale e maggioritario, nel 1995 è forse più corretto parlare di difficoltà a «conquistare pezzi» di elettorato maggioritario. Rispetto al suo calcolo, anzi, la tabella 4, permette ragionevolmente di eliminare la formula dubitativa, dati i saldi - (quasi) sempre inferiori al centro-destra, ma sistematicamente positivi - dell'opposto schieramento. E se questo è vero, si può anche prescindere dalla critica della distribuzione neutrale dell'eccedenza maggioritaria e andare a maggior ragione verso una conclusione che D'Alimonte e Bartolini respingevano sui dati delle politiche. Ora - rettifica infatti D'Alimonte - «quanto abbiamo detto non vuol dire che il centro-sinistra non abbia alcuna possibilità di vincere una quota significativa di collegi nel Nord. Infatti, anche un rendimento negativo, nel senso che abbiamo dato qui a questo termine, potrebbe comunque consentire di ottenere un voto in più dello schieramento rivale ... In altre parole, il cartello elettorale potrebbe essere così ampio, e quindi avere una base di partenza (in voti proporzionali) così elevata, da compensare le defezioni dovute all'indisciplina coalizionale. In tal caso, la somma sarebbe sempre negativa e magari fortemente negativa, ma non comprometterebbe la vittoria».

Prudentemente - finisce D'Alimonte - «questa è una scommessa tutta da verificare» e, a rigore, non si può dargli torto, vista la disomogeneità dei dati sui quali si ragiona e l'ipoteticità dei cartelli futuri. Ma, fatta salva la natura piuttosto artificiosa dell'esercizio, è perlomeno orientativo ricordare che nel 1994 i voti per candidati e liste di sinistra al Nord (Camera) erano, rispettivamente, il 25,7 e il $26,3 \%$, mentre la destra stava al 49,4 e al $44,9 \%$; con le regionali, invece, siamo al 31,8 e al $33,7 \%$ per il centro-sinistra contro il 40 e il $40,4 \%$ del centro-destra ${ }^{35}$;

35 I calcoli tengono conto solo delle regioni nelle quali si è votato nel 1995 ed escludono, quindi, il Trentino-Alto Adige e il Friuli-Venezia Giulia. 
c'è, dunque, un'evidente tendenza al riequilibrio, per di più sicuramente sottostimata se si tiene conto che i tuttora più forti hanno ormai esaurito il loro bacino potenziale di voti, mentre il più debole centro-sinistra può ancora contare su accordi con, ed elettori di, Rifondazione e Lega. A occhio e croce, questo lo porterebbe in vantaggio e, probabilmente, quanto basta per «vincere una quota significativa di collegi», nonostante le (vere o supposte) defezioni «ideologiche»; come dire che la scommessa, forse, è in parte già verificata e una vera «competizione sistemica» e bipolare sul punto di dispiegarsi pienamente.

\section{Conclusioni}

In questo saggio ho condotto un'analisi della transizione italiana palesemente meno «continuista» di molte di quelle oggi in circolazione. Prendendo sul serio i modelli di Sartori, sia dal punto di vista analitico che nell'applicazione empirica, ho potuto infatti dimostrare che né il formato del sistema partitico, né le sue predisposizioni meccaniche si lasciano più spiegare nei termini classici del pluralismo polarizzato: attori rilevanti, distanza ideologica, numero dei poli, e anche il livello di competitività sistemica, sono mutati, o sono in stato di avanzato mutamento. E, tuttavia, mi rendo conto che, se questa conclusione è accettata, segue un'implicazione alquanto paradossale. L'implicazione è che l'Italia è vicina al traguardo del pluralismo moderato, cioè di un tipo di democrazia «stabile» ed «efficiente», nel quale le domande dell'elettorato mediano sono soddisfatte appropriatamente e tempestivamente dalle politiche «moderate» di una classe politica ricettiva, responsabile, accountable. $\mathrm{Ma}-$ ecco il paradosso - molti (me compreso) direbbero che niente di tutto questo è alle viste. E allora?

Allora, si può declinare la responsabilità, come fa Sartori (1982, 206-207) scrivendo che «un modello cerca di individuare i meccanismi ... di una determinata struttura ... E le previsioni del modello sono subordinate», fra l'altro, alla condizione che «la cosiddetta logica di un sistema, e cioè i suoi meccanismi, prevalgano sui suoi operatori. Pertanto, il limite di un modello è presto detto: non può prevedere l'uomo, e più esattamente gli uomini che reagiscono alla meccanica delle strutture e che frenano, o addirittura riescono a prevalere, sui condizionamenti sistemici ... Diciamo, allora, che il mio modello arriva solo fin 
dove arriva l'andamento inerziale di un sistema politico. Prevede un trend, non esiti; e nemmeno può prevedere i tempi. Esiti e tempi dipendono dagli uomini».

Anche se traslata da un diverso scenario, questa citazione fa perfettamente al caso nostro, e non solo perché assolve il modello da demeriti che non ha; ancora più importante è che essa addita - fortuitamente - un anello debole dell'attuale transizione, cioè i comportamenti anacronistici, centrifughi, o imprevedibilmente estemporanei che molti osservatori a ragione imputano a molta parte delle élites italiane ${ }^{36}$.

Così come sono, questi comportamenti rappresentano un'incognita da non sottovalutare, tanto più che essi alimentano il circolo vizioso fra le incertezze della transizione e l'incapacità dei leader di guidarne fuori il paese; ma - aggiungo - l'incognita non va nemmeno sopravvalutata al punto da attribuire alle élites il ruolo chiave, il potere di de- o ri-radicalizzare il sistema con le loro sole scelte o non scelte ed, eventualmente, di generare un «nuovo pluralismo polarizzato» (Morlino 1995, 20-23). Anche se possono modificare i tempi e, dunque, ritardare gli esiti, più difficilmente gli uomini riusciranno, infatti, a manipolare, o addirittura rovesciare, trend strutturali; e sui trend - direbbe comunque Sartori - è «competente» il modello, cioè solo il modello autorizza spiegazioni e predizioni teoricamente giustificate ed empiricamente corroborate.

Nonostante chi rema contro, rimango quindi del parere che, più o meno alla lunga, l'approdo della transizione sia il pluralismo moderato. Ma esso - si potrebbe infine obiettare - non è dietro l'angolo non solo per difetto di uomini, ma anche perché mancano requisiti strutturali di stabilità ed efficienza che sono trascurati dal modello. Vero. Sartori $(1976,291)$ evita, infatti, di estenderlo al sistema elettorale e alla struttura costituzionale, pur ammettendo che l'inclusione di queste «variabili intervenienti» ne aumenterebbe l'accuratezza ed il potere predittivo. Concordo con Morlino e Tarchi (1995), inoltre, che i drammatici livelli di insoddisfazione dei cittadini additano due altri aspetti (inefficienza della pubblica amministrazione e crisi dello Stato sociale e interventista) che io stesso mettevo fra le componenti della transizione all'inizio di questo lavoro. Senza dubbio, queste sono materie sulle quali si è fatto poco o nulla, a parte

36 D'Alimonte e Bartolini (1995, 457); Morlino (1995, 20-23); Pasquino (1995); Segatti (1995). 
l'introduzione, incisiva, ma parzialmente efficace, del maggioritario e alcune scelte di risanamento (riforma delle pensioni, privatizzazioni) volte ad arginare le tendenze più esplosive dell'emergenza finanziaria. Le «variabili intervenienti», dunque, sono parecchie e, purtroppo, più o meno mal raccordate da un punto di vista teorico. Attualmente, infatti, la modellistica dei sistemi elettorali coesiste fianco a fianco con la ricerca sui sistemi elettorali e sull'ingegneria costituzionale, altrettanto sviluppata, ma non ancora fusa sistematicamente con la prima, nonostante contributi assai importanti (Fisichella 1982; Lijphart 1994). Le molte teorie dell' «implementazione», dal canto loro, mancano del tutto o fanno riferimenti solo embrionali e frammentari ai rapporti fra sistema politico-istituzionale e subsistema amministrativo. E la schiera fin troppo folta degli studiosi della crisi dello Stato sociale continua ad accumulare framework, interpretazioni e modelli economici, sociologici, politologici, che vanno per lo più per conto proprio entro e fra settori disciplinari.

Di fronte a questa intrattabile e confusa complessità, mi è sembrato indispensabile scegliere la parsimonia e la rilevanza, mettendo rigorosamente alla prova il modello più classico ed autorevole disponibile sulla democrazia italiana. I risultati - credo - sono eloquenti, anche se certo non esaurienti. Estensioni ed integrazioni, pertanto, sono benvenute, specie se in grado di contribuire cumulativamente alla spiegazione scientifica della transizione.

\section{Riferimenti bibliografici}

Bardi, L. (1996), Party System Change and Anti-Party Sentiments in Italy. Forza Italia, The Lega Nord and Other Things, in «European Journal of Political Research», di prossima pubblicazione.

Bartolini, S. (1986), La volatilità elettorale, in «Rivista Italiana di Scienza Politica», XVI, n. 3, pp. 363-400.

- (1995), Electoral Competition: Analytical Dimensions and Empirical Problems, relazione al Symposium on Political Parties, Madrid, Instituto Juan March.

Bartolini, S. e R. D'Alimonte (a cura di) (1995), Maggioritario ma non troppo. Le elezioni politiche del 1994, Bologna, Il Mulino.

Bartolini, S. e R. D'Alimonte (1995), La competizione maggioritaria: Le origini elettorali del parlamento diviso, in S. Bartolini e R. D'Alimonte (a cura di), pp. 317-372.

Bartolini, S. e P. Mair, (1990), Identity, Competition, and Electoral 
Availability. The Stabilization of European Electorates 1885-1985, Cambridge, Cambridge University Press.

Biorcio, R. e P. Natale (1989), La mobilità elettorale degli anni ottanta, in «Rivista Italiana di Scienza Politica», XIX, n. 3, pp. 385-430.

Calvi, G. e A. Vannucci (1995), L'elettore sconosciuto. Analisi socioculturale e segmentazione degli orientamenti politici nel 1994, Bologna, Il Mulino.

Cartocci, R. (1994), Fra Lega e Chiesa. L'Italia in cerca di interpretazione, Bologna, Il Mulino.

Ceccanti, S. e S. Fabbrini (1995), Transizione verso Westminster? Ambiguità e discontinuità nella formazione del governo Berlusconi, in G. Pasquino (a cura di), pp. 257-284.

Chiaramonte, A. (1995), Gli effetti distorsivi del nuovo sistema elettorale, in S. Bartolini e R. D'Alimonte (a cura di), pp. 373-400.

Comiskey, M. (1993), Electoral Competition and the Growth of Public Spending in 13 Industrial Democracies, 1950 to 1983, in «Comparative Political Studies», XXVI, n. 3, pp. 350-374.

Corbetta P., A.M.L. Parisi e H.M.A. Schadee (1988), Elezioni in Italia. Struttura e tipologia delle consultazioni politiche, Bologna, Il Mulino.

D'Alimonte, R. (1978), Competizione elettorale e rendimento politico: il caso italiano, in «Rivista Italiana di Scienza Politica», VIII, n. 3 , pp. 457-493.

- (1989), Democrazia e competizione, in «Rivista Italiana di Scienza Politica», XIX, n. 2, pp. 301-319.

- (1995), La transizione italiana: Il voto regionale del 23 aprile, in «Rivista Italiana di Scienza Politica», XXV, n. 3, pp. 515-558.

D'Alimonte R. e S. Bartolini (1995), Il sistema partitico: una transizione difficile, in S. Bartolini e R. D'Alimonte (a cura di), pp. 427 466.

Diamanti, I. (1995), La Lega. Geografia, storia e sociologia di un soggetto politico, Roma, Donzelli Editore, $2^{a}$ ed.

Diamanti, I. e R. Mannheimer (a cura di) (1994), Milano a Roma. Guida all'Italia elettorale del 1994, Roma, Donzelli Editore.

Di Virgilio, A. (1995), Dai partiti ai poli: la politica delle alleanze, in S. Bartolini e R. D’Alimonte (a cura di), pp. 177-232.

Eijk, van Der C. e E.V. Oppenhuis (1991), 'European Parties' Performance in Electoral Competition, in «European Journal of Political Research», XIX, n. 1, pp. 55-80.

Fisichella, D. (1982), Elezioni e democrazia. Un'analisi comparata, Bologna, Il Mulino.

Galli, G. (1966), Il bipartitismo imperfetto, Bologna, Il Mulino.

Gunther, R. e J.R. Montero (1994), Los Anclajes del Partidismo: un análisis comparado del comportamiento electoral en cuatro democracias del sur de Europa, in P. del Castillo (a cura di), Comportamiento Político y Electoral, Madrid, CIS, pp. 467-520. 
Ignazi, P. (1995), Postfascisti? Dal Movimento Sociale ad Alleanza Nazionale, Bologna, Il Mulino.

Ignazi, P. e R.S. Katz (a cura di) (1995), Politica in Italia. Edizione 1995, Bologna, Il Mulino.

Lijphart, A. (1968), The Politics of Accommodation. Pluralism and Democracy in the Netherlands, Berkeley, University of California Press.

- (1968a), Typologies of Democratic Systems, in «Comparative Political Studies», I, n. 1, pp. 3-44.

- (1984), Democracies. Patterns of Majoritarian and Consensus Government in Twenty-One Countries, New Haven, Yale University Press.

- (1994), Electoral Systems and Party Systems. A Study of Twenty-Seven Democracies, 1945-1990, Oxford, Oxford University Press.

Mannheimer, R. (1986), Come spiegare la mobilità elettorale in Italia, in «Rivista Italiana di Scienza Politica», XVI, n. 1, pp. 46-80.

Maraffi, M. (1995), Forza Italia, in G. Pasquino (a cura di), La politica italiana. Dizionario critico 1945-95, Roma-Bari, Laterza, pp. $247-$ 259.

Massari, O. (1995), La selezione dei candidati, in G. Pasquino (a cura di), pp. 21-48.

Mattina, L. (1995), I candidati, in S. Bartolini e R. D'Alimonte (a cura di), pp. 233-270.

Melchionda, E. (1995), Il bipartitismo irrealizzato. Modelli di competizione nei collegi uninominali, in G. Pasquino (a cura di), pp. 131207.

Morlino, L. (1995), Crisis of Parties and Change of Party System in Italy, in «Party Politics», di prossima pubblicazione.

Morlino, L. e J.R. Montero, (1994), Legittimità, consolidamento e crisi nell'Europa meridionale, in «Rivista Italiana di Scienza Politica», XXIV, n. 1, pp. 27-66.

Morlino, L. e M. Tarchi (1995), The Dissatisfied Society: The Roots of Political Change in Italy, in «European Journal of Political Research», di prossima pubblicazione.

Norris, P. e I. Crewe (1994), Did the British Marginals Vanish? Proportionality and Exaggeration in the British Electoral System Revisited, in «Electoral Studies», XIII, n. 3, pp. 201-221.

Pappalardo, A. (1989), L'analisi economica della politica, in A. Panebianco (a cura di), L'analisi della politica, Bologna, Il Mulino, pp. 193-216.

Pasquino, G. (1994), Shaping a Better Republic? The Italian Case in a Comparative Perspective, Working Paper n. 62, Madrid, Instituto Juan March.

- (1995), Un sistema politico che cambia. Transizione o restaurazio$n e$ ?, in G. Pasquino (a cura di), pp. V-XV.

Pasquino G. (a cura di) (1995), L'alternanza inattesa. Le elezioni del 27 marzo 1994 e le loro conseguenze, Soveria Mannelli, Rubettino. 
Przeworski, A. (1975), Institutionalization of Voting Pattern or Is Mobilization the Source of Decay?, in «American Political Science Review», LXIX, n. 1, pp. 49-67.

Ricolfi, L. (1995), Il voto proporzionale: il nuovo spazio politico italia$n o$, in S. Bartolini e R. D'Alimonte (a cura di), pp. 273-316.

Sani, G. e G. Sartori (1978), Frammentazione, polarizzazione e cleavagers: democrazie facili e difficili, in «Rivista Italiana di Scienza Politica», VIII, n. 3, pp. 339-362.

Sartori, G. (1976), Parties and Party Systems. A Framework for Analysis, Cambridge, Cambridge University Press.

- (1977), Democrazia competitiva e élites politiche, in «Rivista Italiana di Scienza Politica», VII, n. 3, pp. 327-356.

- (1982), Teoria dei partiti e caso italiano, Milano, Sugarco Edizioni.

Segatti, P. (1995), I programmi elettorali e il ruolo dei mass media, in S. Bartolini e R. D'Alimonte (a cura di), pp. 147-173.

Vassallo, S. (1995), La politica delle coalizioni. Da un sistema partitico all'altro, in G. Pasquino (a cura di), pp. 49-93. 